US Army Corps of Engineers ${ }_{\circledast}$

Engineer Research and

Development Center

\title{
FRDC
}

Materials and Modeling for Force Protection

\section{Synthesis and Characterization of Biological Nanomaterial/Poly(vinylidene fluoride) Composites}

Eftihia Barnes, Jennifer A. Jefcoat, Erik M. Alberts, Hannah R.

Peel, L. Christopher Mimun, J. Paige Buchanan, Xin Guan,

Christopher M. Warner, Kyle L. Klaus, Erin R. Reed-Gore, and Charles A. Weiss Jr.

\section{0, 1, 2, 4,8 wt\% CeNF/PVDF}
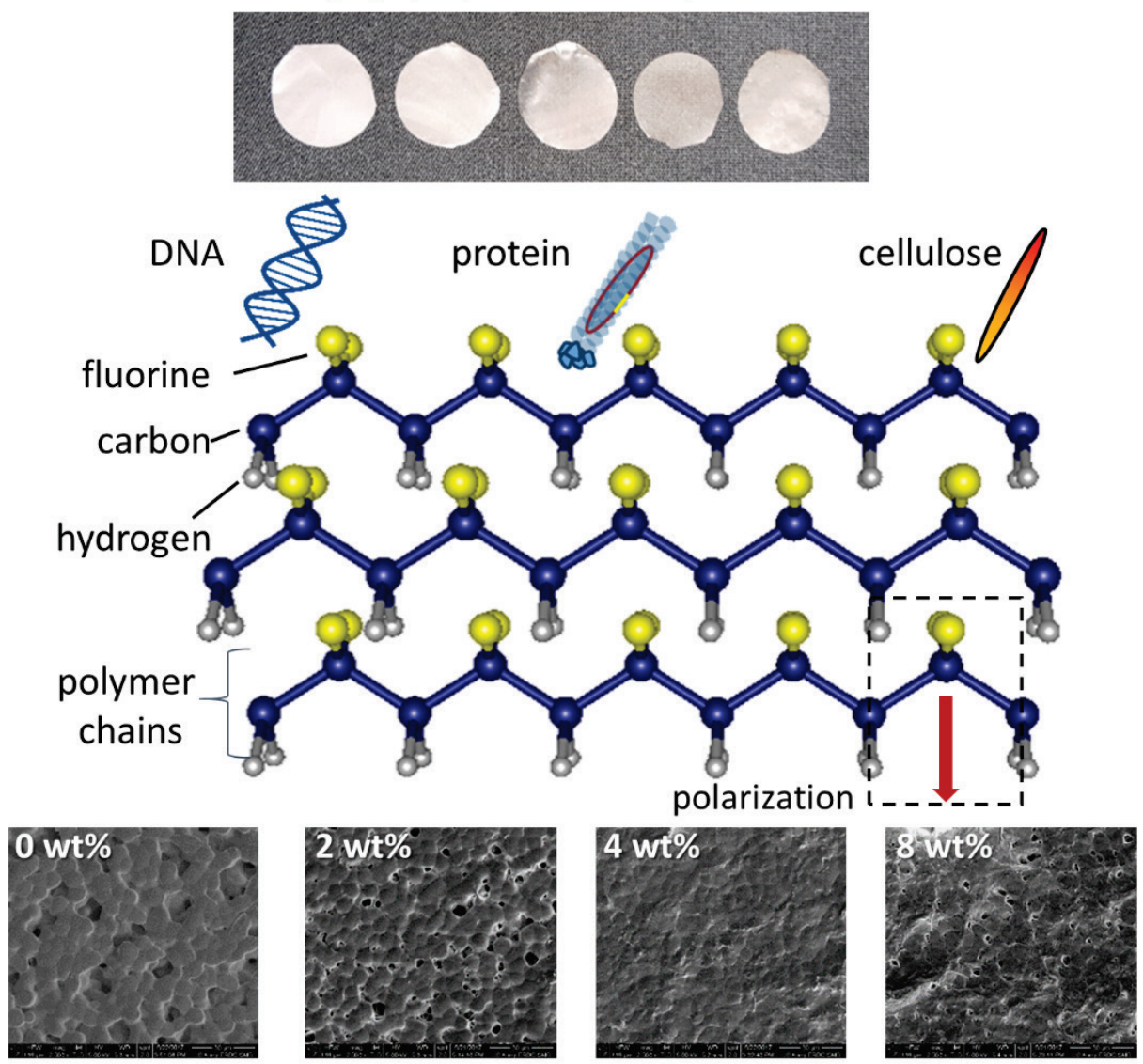
The U.S. Army Engineer Research and Development Center (ERDC) solves the nation's toughest engineering and environmental challenges. ERDC develops innovative solutions in civil and military engineering, geospatial sciences, water resources, and environmental sciences for the Army, the Department of Defense, civilian agencies, and our nation's public good. Find out more at www.erdc.usace.army.mil.

To search for other technical reports published by ERDC, visit the ERDC online library at https://erdclibrary.on.worldcat.org/discovery. 


\section{Synthesis and Characterization of Biological Nanomaterial/Poly(vinylidene fluoride) Composites}

Eftihia Barnes, Jennifer A. Jefcoat, J. Paige Buchanan, Kyle L. Klaus, Erin R. Reed-Gore, and Charles A. Weiss Jr.

Geotechnical and Structures Laboratory

U.S. Army Engineer Research and Development Center

3909 Halls Ferry Road

Vicksburg, MS 39180-6199

Hannah R. Peel, L. Christopher Mimun, and Christopher M. Warner

Environmental Laboratory

U.S. Army Engineer Research and Development Center

3909 Halls Ferry Road

Vicksburg, MS 39180-6199

Erik M. Alberts

HX5 LLC

3530 Manor Drive

Vicksburg, MS 39180-5625

Xin Guan

Bennett Aerospace

1100 Crescent Green, Suite 250

Cary, NC 27511-4470

Final report

Approved for public release; distribution is unlimited.

Prepared for Office of the Assistant Secretary of the Army Acquisition, Logistics and Technology

Washington, DC 20310-0103

Under 62784/T40/Materials and Modeling for Force Protection, "Synthesis and Characterization of Flexible Polymer Composites with Biological Material Enabled Piezoelectricity" 


\section{Abstract}

The properties of composite materials are strongly influenced by both the physical and chemical properties of their individual constituents, as well as the interactions between them. For nanocomposites, the incorporation of nano-sized dopants inside a host material matrix can lead to significant improvements in mechanical strength, toughness, thermal or electrical conductivity, etc. In this work, the effect of cellulose nanofibrils on the structure and mechanical properties of cellulose nanofibril poly(vinylidene fluoride) (PVDF) composite films was investigated. Cellulose is one of the most abundant organic polymers with superior mechanical properties and readily functionalized surfaces. Under the current processing conditions, cellulose nanofibrils, as-received and 2,2,6,6-tetramethylpiperidine-1-oxyl radical (TEMPO) oxidized, alter the crystallinity and mechanical properties of the composite films while not inducing a crystalline phase transformation on the $\gamma$ phase PVDF composites. Composite films obtained from hydrated cellulose nanofibrils remain in a majority $\gamma$ phase, but also exhibit a small, yet detectable fraction of $\alpha$ and $\beta$ PVDF phases.

DISCLAIMER: The contents of this report are not to be used for advertising, publication, or promotional purposes. Citation of trade names does not constitute an official endorsement or approval of the use of such commercial products. All product names and trademarks cited are the property of their respective owners. The findings of this report are not to be construed as an official Department of the Army position unless so designated by other authorized documents. 


\section{Contents}

Abstract.............................................................................................................................. ii

Figures and Tables.................................................................................................iv

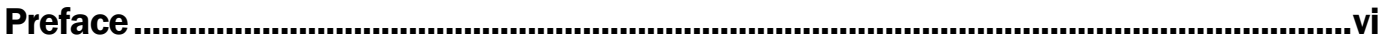

1 Introduction ........................................................................................................... 1

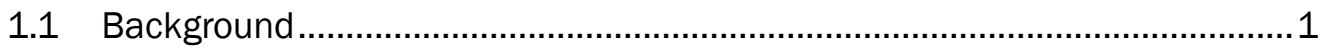

1.2 Objectives..................................................................................... 4

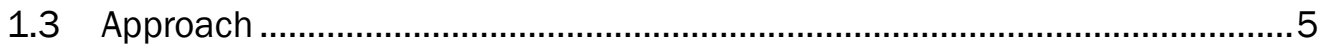

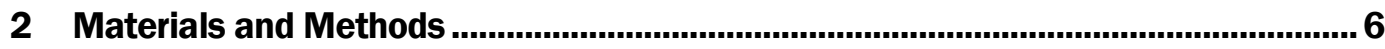

2.1 Materials and synthesis of composite films ..........................................6 6

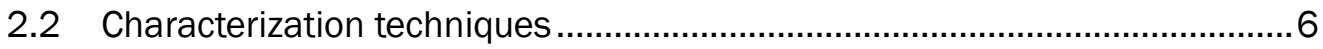

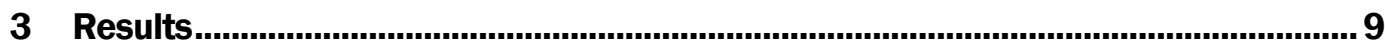

4 Conclusions and Recommendations....................................................................22

References.......................................................................................................24

Appendix A: PVDF Films with Added Water.............................................................29

Appendix B: Cellulose Nanofibril/PVDF Films Obtained from As-Received

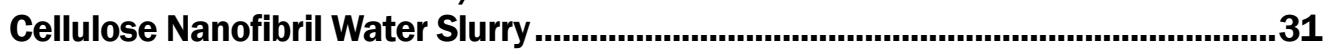

Appendix C: Melting and Recrystallization of Cellulose Nanofibril/PVDF

Composites - Study 1................................................................................................40

Appendix D: Melting and Recrystallization of Cellulose Nanofibril/PVDF

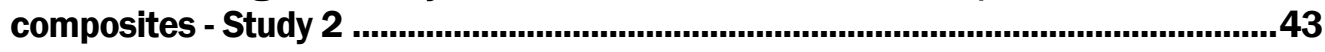

Appendix E: DNA/PVDF Composites .............................................................................44

Symbols and Acronyms ...................................................................................46

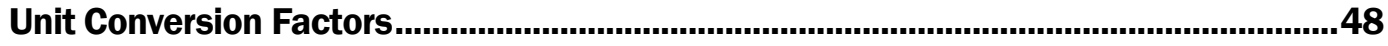

Report Documentation Page 


\section{Figures and Tables}

\section{Figures}

Figure 1. PVDF chain conformations for the A. $\alpha$, B. $\beta$, and C. $\gamma$ PVDF crystalline phases. The arrows show the direction of electrical dipole. Carbon is denoted by "C," fluorine with "F," and hydrogen with "H.".

Figure 2. FTIR spectra of $\alpha, \beta$, and $\gamma$ PVDF with labeled characteristic bands. Adapted from Martins et al. (2014).

Figure 3. Characterization of cellulose. A. SEM image showing high-aspect ratio fibrils of the as-received cellulose. B. AFM scan revealing cellulose nanocrystals, in addition to cellulose nanofibrils. C. AFM of TEMPO-mediated oxidized cellulose revealing the presence of finer particles after the TEMPO treatment. AFM scan sizes are $5 \mu \mathrm{m} \times 5 \mu \mathrm{m}$.

Figure 4. FTIR spectra of as-received and TEMPO-mediated oxidized cellulose.

Figure 5. SEM images of the film surface for A. Neat PVDF, B. $0.5 w t \%$, C. 1 wt $\%$, D. 2 wt\%, E. 3 wt\%, F. 4 wt\% and G. 5 wt\% cellulose nanofibril/PVDF composites (series A).

Figure 6. SEM images of the film surface for A. 0.5 wt\%, B. 1 wt\%, C. 2 wt\%, D. 3 wt\%, E. 4 wt\% and F. 5 wt\% oxidized cellulose nanofibril/PVDF composites (series B).

Figure 7. XRD patterns for A. As-received cellulose nanofibril/PVDF composites (series A); and B. Oxidized cellulose nanofibril/PVDF composites (series B).

Figure 8. A. FTIR spectra of neat PVDF and as-received cellulose nanofibril/PVDF composites (series A). B. Calculated intensity ratios for qualitative estimation of $\alpha, \beta$, and $\gamma$ phases in the composite films as function of cellulose wt $\%$.

Figure 9. A. FTIR spectra of neat PVDF and oxidized cellulose nanofibril/PVDF composites (series B). B. Calculated intensity ratios for qualitative estimation of $\alpha, \beta$, and $\gamma$ phases in the composite films as function of oxidized cellulose nanofibril wt\%.

Figure 10. FIIR spectra in the $3100-3600 \mathrm{~cm}^{-1}$ range for $A$. as-received (series A), and B. oxidized cellulose nanofibril/PVDF composites (series B). No hydroxyl peaks were observed for either film series.

Figure 11. DSC showing the melting behavior of the neat PVDF, and A. asreceived (series $A$ ), B. oxidized cellulose nanofibril/PVDF composites (series $B$ ).

Figure A1. A. FIIR spectra of neat PVDF and PVDF films with water added to the solvent (series C). B. FTIR spectra in the $3100-3600 \mathrm{~cm}^{-1}$ range showing the lack of the hydroxyl band. C. Calculated intensity ratios for qualitative estimation of $\alpha$, $\beta$, and $\gamma$ phases in the composite films as function of added water wt\%.

Figure B1. A. Picture of 0, 1, 2, 4 and 8 wt\% cellulose nanofibril/PVDF films (series D). Increasing cellulose nanofibril wt\% from left to right. $20 \times$ magnification optical crossed-polarized images of B. 0 wt $\%$, C. 1 wt \%, D. 2 wt\%, E. $4 \mathrm{wt} \%$, and F. 8 wt\% cellulose nanofibril/PVDF composites (series D).

Figure B2. SEM images of film surface for A. Neat PVDF, B. 1 wt\%, C. 2 wt\%, D. 4 wt\%, and E. 8 wt\% cellulose nanofibril/PVDF composites (series D). 
Figure B3. XRD patterns for cellulose nanofibril/PVDF composites (series D).

Figure B4. A. FTIR spectra of neat PVDF and cellulose nanofibril/PVDF composites (series D). B. Calculated intensity ratios for qualitative estimation of $\alpha, \beta$, and $\gamma$ phases in the composite films as function of oxidized cellulose $\mathrm{wt} \%$.

Figure B5. FTIR spectra of neat PVDF and cellulose nanofibril/PVDF composites (series D) A. Spectra in the 3100-3600 $\mathrm{cm}^{-1}$ range showing the emergence of the hydroxyl band with increasing cellulose wt $\%$. B. Spectra in the $2940-3060 \mathrm{~cm}^{-1}$ range showing $\mathrm{CH}_{2}$ vibrational peaks as function of cellulose wt\%.

Figure B6. A. High-resolution SEM image showing the surface morphology of the $4 \mathrm{wt} \%$ composite film, which consists of at least two morphologically distinct phases. B. Micro Raman spectra collected at locations similar to the ones shown in A ("mainly $\alpha$ and $\gamma$ " and "mainly $\gamma$ and $\alpha$ " spectra). The spectrum labeled "mainly $\beta$ and $\alpha$ " was obtained from a commercial piezoelectric PVDF film.

Figure B7. DSC showing the melting behavior of the neat PVDF, and the asreceived cellulose nanofibril/PVDF composites (series D).

Figure C1. 20× magnification optical crossed-polarized images of A. 0 wt\%, B. 1 wt $\%$, C. 2 wt $\%$, D. 4 wt\%, and E. 8 wt\% cellulose nanofibril/PVDF composites (series D) after thermal treatment at $200^{\circ} \mathrm{C}$.

Figure C2. SEM images of the film surface for A. Neat PVDF, B. 1 wt $\%$, C. 2 wt $\%$, D. 4 wt\%, and E. 8 wt\% cellulose nanofibril/PVDF composites (series D) after thermal treatment at $200^{\circ} \mathrm{C}$.

Figure C3. FIIR spectra of neat PVDF and cellulose nanofibril/PVDF composites (series D) after thermal treatment at $200^{\circ} \mathrm{C}$.

Figure D1. FTIR spectra of A. neat PVDF films, B. 5 wt\% cellulose nanofibril/PVDF composites as function of melting temperature.

Figure E1. FTIR spectra of neat PVDF, 0.25 wt $\%$ and 1.5 wt\% DNA/PVDF, and 2 wt\% commercial DNA/PVDF composites.

\section{Tables}

Table 1. FTIR bands characteristic of $\alpha, \beta$ and $\gamma$ PVDF (Martins et al. 2014).

Table 2. Summary of experimental processing conditions and films series studied in this report.

Table 3. FTIR crystalline phase fraction and DSC results for as-received cellulose nanofibril/PVDF films (series A) and oxidized cellulose nanofibril/PVDF films (series B).

Table 4. Tensile properties of as-received cellulose nanofibril/PVDF films (Series A) and oxidized cellulose nanofibril/PVDF films (Series B).

Table B1. FTIR crystalline phase fraction and DSC results for as-received cellulose nanofibril/PVDF films (Series D).

Table B2. Tensile properties of as-received cellulose nanofibril/PVDF films (Series D). 


\section{Preface}

This study was conducted for Office of the Assistant Secretary of the Army Acquisition, Logistics and Technology, Washington, DC, 20310-0103 under 62784/T40/Materials and Modeling for Force Protection, "Synthesis and Characterization of Flexible Polymer Composites with Biological Material Enabled Piezoelectricity." The technical monitor was Ms. Pamela G. Kinnebrew, Lead Technical Director for Military Engineering, Geotechnical and Structures Laboratory.

The work was performed by the Structural Mechanics Branch (GSM) of the Geosciences and Structures Division (GS) and the Concrete and Materials Branch (GMC) of the Engineering Systems and Materials Division (GM), U.S. Army Engineer Research and Development Center, Geotechnical and Structures Laboratory (ERDC-GSL), and the Environmental Chemistry Branch (EPC) and the Environmental Processes Branch (EPP) of the Environmental Processes and Engineering Division (EP), ERDC Environmental Laboratory (ERDC-EL). At the time of publication, Mr. Bradford A. Steed was Chief, GSM; Dr. Jameson Shannon was Chief, GMC; Ms. Amber L. Russell was Chief, EPC; Dr. Brandon J. Lafferty was Chief, EPP; Mr. James L. Davis was Chief, GS; Mr. Justin S. Strickler was Chief, CEERD-GM; Mr. Warren P. Lorentz was Chief, EP; and Ms. Pamela G. Kinnebrew, CEERD-GZT; was the Lead Technical Director for Military Engineering, Survivability and Protective Structures. The Deputy Director of ERDC-GSL was Mr. Charles W. Ertle II, and the Director was Mr. Bartley P. Durst. The Deputy Director of ERDC-EL was Mr. Jack E. Davis, and the Director was Mr. Ilker R. Adiguzel.

COL Teresa A. Schlosser was the Commander of ERDC, and Dr. David W. Pittman was the Director. 


\section{Introduction}

\subsection{Background}

Materials whose properties change in response to environmental, physical, or chemical stimuli are of immense scientific and technological interest due to both the underlying physics, chemistry, and structure/property relationships, as well as their potential applications in future technologies. Among smart polymers, poly(vinylidene fluoride) (PVDF) exhibits outstanding mechanical and electrical properties, such as ferroelectricity, pyroelectricity and piezoelectricity. PVDF has been used as the building block of smart materials including actuators (Perez et al. 2012), transducers (Bar-Cohen and Zhang 2008), sensors (Sirohi and Chopra 2000; Ying et al. 2002), nonvolatile memory components (Ouyang et al. 2004; Scott and Bozano 2007), energy harvesters (Chang et al. 2010; Beeby et al. 2006 and 2007; Lee et al. 2012), etc. When compared to single crystals and ceramics, polymer-based piezoelectrics have high strength, and high-impact resistance; are lightweight, tough, and flexible; and their manufacturing is scalable and relatively low cost (Ramadan et al. 2014).

PVDF is a semi-crystalline polymer with five known crystalline phases $(\alpha, \beta$, $\gamma, \delta$, and $\varepsilon$ ) whose formation is highly dependent on processing conditions (Martins et al. 2014). The most common phases are the non-polar, nonelectroactive $\alpha$ phase with antiparallel TGTG' (trans-gauche-trans-gauche) chain conformation, and the electroactive $\beta$ and $\gamma$ phases with all trans (TTTT) planar and $\mathrm{T}_{3} \mathrm{GT}_{3} \mathrm{G}^{\prime}$ chain conformations, respectively (Weinhold et al. 1980; Lovinger 1981; Martins et al. 2014). In the $\beta$ phase, the fluorine anions are located opposite from the hydrogen cations maximizing polarizability and enabling the development of macroscopic polarization. On the contrary, the presence of the $\mathrm{G}^{\prime}$ gauche bond in every fourth repeat unit reduces the net polarization of the $\gamma$ phase. The chain conformations of the $\alpha, \beta$, and $\gamma$ phases and direction of local electrical dipoles (polarization) are shown in Figure 1. For electroactive applications, highly crystalline films with majority $\beta$ or $\gamma$ phases are desired.

In general, the $\alpha$ phase can be easily distinguished from the $\beta$ and $\gamma$ phases by employing common materials characterization techniques such as FTIR and Raman spectroscopy, XRD, and DSC. An excellent review journal publication on PVDF crystalline phases, effect of processing conditions, 
and their experimental identification is by Martins et al. (2014).

Unambiguous identification of the $\beta$ and $\gamma$ phases is a challenging task, with incorrect phases reported in much of the published literature. Here, the FTIR spectra of PVDF will be discussed in detail, because FTIR spectroscopy is a quick and reliable method for distinguishing the $\alpha, \beta$, and $\gamma$ phases (Milani et al. 2015). Representative FTIR absorption bands from the three phases are listed in Table 1 (Martins et al. 2014). The absorption bands located at $510(\beta)$ and $512(\gamma) \mathrm{cm}^{-1}$ are spectrally very close, whereas the band at $840 \mathrm{~cm}^{-1}$ is common to both phases. Hence, these bands are not optimum markers for unambiguous identification of the $\beta$ or $\gamma$ phase. On the contrary, the peak at $1276 \mathrm{~cm}^{-1}$ is unique to the $\beta$ phase, whereas the peak at $1232 \mathrm{~cm}^{-1}$ is unique to the $\gamma$ phase. Figure 2 shows representative FTIR spectra of $\alpha, \beta$, and $\gamma$ PVDF.

Figure 1. PVDF chain conformations for the A. $\alpha$, B. $\beta$, and C. $\gamma$ PVDF crystalline phases. The arrows show the direction of electrical dipole. Carbon is denoted by " $\mathrm{C}$," fluorine with "F," and hydrogen with " $\mathrm{H}$. "

A
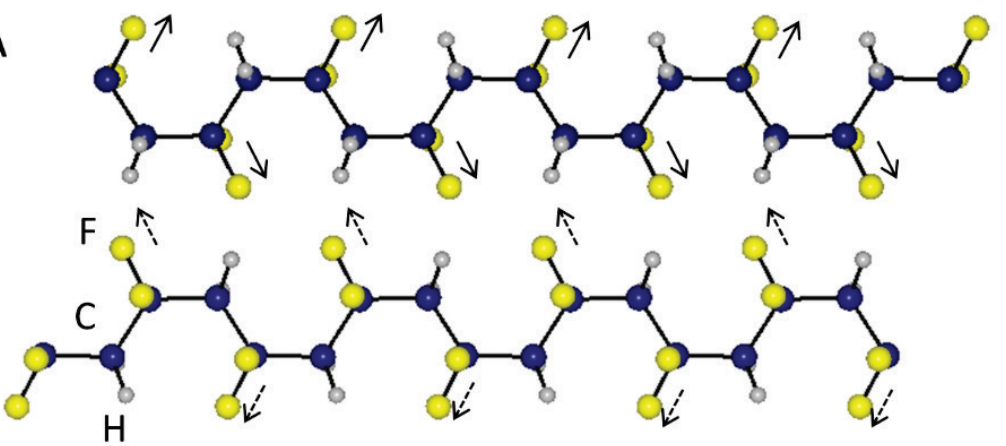

B

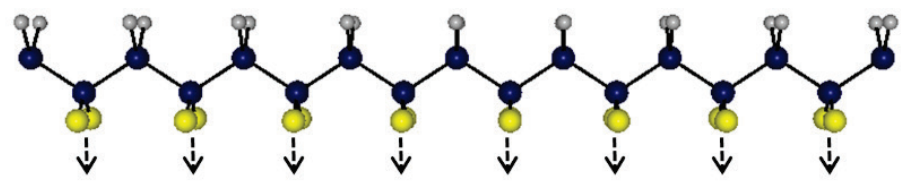

C

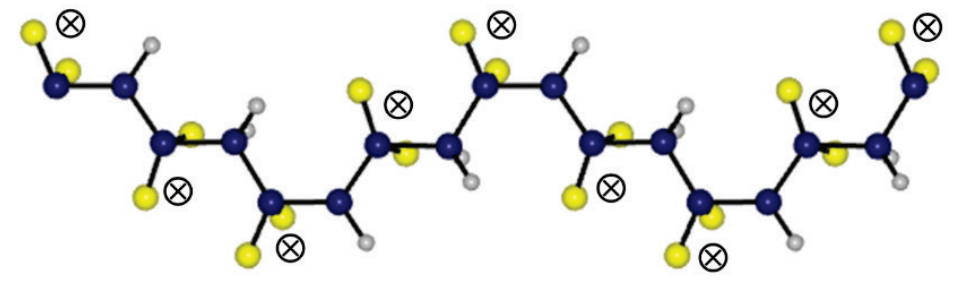


Figure 2. FTIR spectra of $\alpha, \beta$, and $\gamma$ PVDF with labeled characteristic bands. Adapted from Martins et al. (2014).

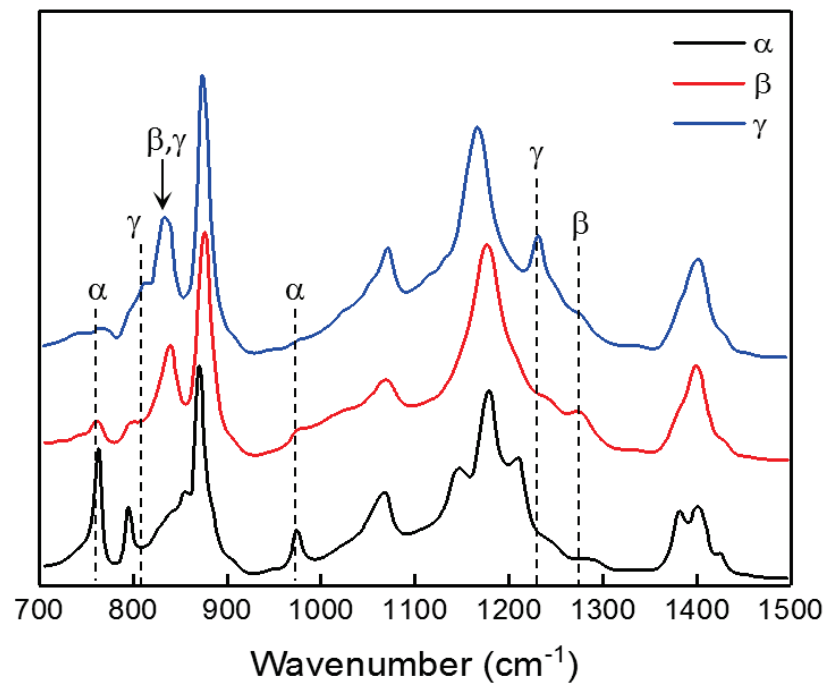

Table 1. FIIR bands characteristic of $\alpha, \beta$ and $\gamma$ PVDF (Martins et al. 2014).

\begin{tabular}{|l|l|l|}
\hline$\alpha\left(\mathrm{cm}^{-1}\right)$ & $\beta\left(\mathrm{cm}^{-1}\right)$ & $\gamma\left(\mathrm{cm}^{-1}\right)$ \\
\hline 408 & 510 & 431 \\
532 & 840 & 512 \\
614 & 1276 & 776 \\
766 & & 811 \\
795 & & 833 \\
855 & & 840 \\
976 & & 1232 \\
\hline
\end{tabular}

Numerous processing methods have been employed to induce polar phase formation in PVDF. Starting from $\alpha$ PVDF, the $\beta$ phase can be obtained by mechanical stretching (Salimi and Youse 2003; Sencadas et al. 2009), or from the $\alpha$ melt under high pressure (Doll and Lando 1970; Pan et al. 2012; Hattori et al. 1996), fast cooling (Yang and Thomas 1987; Gradys et al. 2007), external electric field application (Zheng et al. 2007; Ribeiro et al. 2010), or the addition of fillers, such as dielectric and metallic nanoparticles (Ye et al. 2013; Mandal et al. 2012; Wang et al. 2011; Sencadas et al. 2011), clays (Patro et al. 2008), carbon nanomaterials (Yu et al. 2009; Costa et al. 2009; El Achaby et al. 2013), hygroscopic salts (Yoon et al. 2008), and other polymers (Yang and Chen 1987). Low temperature crystallization of PVDF solutions with DMF and DMSO leads to the formation of predominantly $\gamma$ phase PVDF (Benz et al. 2002), as does prolonged high temperature, thermal annealing of the $\alpha$ phase (Gregorio and Capitao 2000), or addition of certain additives (Lopes et al. 2011 and 2012). Dopant-induced $\gamma$ to $\beta$ 
PVDF transformation is not very common but it has been reported by Benz et al. (2002) and Tamang et al. (2015). In Tamang et al. (2015), the incorporation of deoxyribonucleic acid (DNA) resulted in the formation of a film with a highly orientated $\beta$ phase. In addition to controlling PVDF phase formation, dopants can be further used to modify mechanical, rheological, and thermal properties of the composites.

In recent years, there has been a growing interest in abundant and environmentally friendly materials that can be used as additives in functional polymers such as PVDF. One of these additives is cellulose, which is the most abundant organic polymer and the basic structural building block of plants and biofilms secreted from certain types of bacteria (Moon et al. 2011). Cellulose consists of a linear chain of ringed glucose molecules with a flat, ribbon-like configuration. Cellulose assembles into cellulose nanocrystals that are crystalline, rod-shaped particles, which further assemble into cellulose nanofibrils that consist of crystalline regions connected to each other via amorphous cellulose regions.

\subsection{Objectives}

Recent studies on cellulose/PVDF composites claim that incorporation of cellulose promotes $\beta$ phase formation due to charged interactions between the hydroxyl (-OH) groups on the cellulose surface and the fluorine atoms of PVDF (Bodkhe et al. 2014; Rajesh et al. 2014). In another study, incorporation of cellulose nanocrystals (CNCs) in electrospun PVDF increased both the relative fraction of the $\beta$ phase and the crystallinity of the composite fibers over those of neat fibers (Fu et al. 2017). A recent study on electrospun PVDF/cellulose fibers concluded that addition of medium size cellulose has no effect on the formation of $\beta$ phase (Issa et al. 2016). Further, in Rajesh et al. (2014), cellulose nanowhiskers were added to PVDF in the form of a water suspension, but no information was given regarding the amount of solids in the suspension. In addition, the FTIR spectra shown in the particular paper span from 450 to $1000 \mathrm{~cm}^{-1}$, thereby excluding the $1276 \mathrm{~cm}^{-1}$ peak, which is unique to the $\beta$ phase. Finally, the presented XRD patterns from the composite films do not conclusively distinguish the $\beta$ and $\gamma$ phases. Similar experimental deficiencies are found in the paper by Bodkhe et al. (2014). However, the addition of cellulose in neat PVDF does lead to the creation of composites with a higher content of polar phase. In light of these experimental deficiencies and varying results, further investigation is needed in order to elucidate (1) piezoelectric phase formation in PVDF 
due to the incorporation of cellulose nanofibrils, and (2) the mechanisms responsible for the formation of such phases. In this report, the effect of processing conditions on the physical and mechanical properties of cellulose nanofibril/PVDF composite films was investigated. As part of this project, the effect of DNA on the structure of DNA/PVDF composite films, in an attempt to recreate the experimental results of Tamang et al. (2015), was also investigated. These results are shown in Appendix E.

\subsection{Approach}

Commercially available cellulose and TEMPO-mediated oxidized cellulose were incorporated into PVDF. Four series of films were studied: series A was obtained with anhydrous DMF and solvent-exchanged cellulose; series B was obtained with anhydrous DMF and solvent-exchanged TEMPO mediated oxidized cellulose; series $\mathrm{C}$ was obtained with anhydrous DMF, with added water; and series D was obtained from distilled reagent grade DFM and asreceived cellulose (no solvent exchange).The highest cellulose wt\% for series $\mathrm{A}, \mathrm{B}$, and $\mathrm{C}$ was $5 \mathrm{wt} \%$, whereas for series $\mathrm{D}$ it was $6 \mathrm{wt} \%$. The composite films were then characterized with various materials characterization techniques including XRD, FTIR, Raman, DSC, TGA. The tensile properties of the composites from Series A and B were also evaluated. 


\section{Materials and Methods}

\subsection{Materials and synthesis of composite films}

Cellulose nanofibril/PVDF films were prepared by dissolving PVDF pellets (Sigma Aldrich 427152, MW 180,000) in anhydrous DMF (Sigma Aldrich 1023752500 ) at $60^{\circ} \mathrm{C}$ to obtain a $10 \mathrm{wt} \%$ solution, and then adding a known amount of cellulose. Wood pulp cellulose nanofibril aqueous slurry ( $\sim 4 \mathrm{wt} \%$ solids, 96 wt\% fines) was obtained from the University of Maine Process Development Center. For sample series A, B, and D, the aqueous slurry was first solvent-exchanged in DMF and then incorporated into the PVDF/DMF mixture. The wt\% reported here is that of the cellulose solids. Films were cast onto clean glass plates using a \#30 casting blade, and solvent evaporation was achieved at $100^{\circ} \mathrm{C}$ overnight. TEMPO (Sigma Aldrich 214000) oxidized cellulose nanofibril/PVDF films (series B) were also synthesized. Briefly, oxidized cellulose was obtained by adding TEMPO (0.06 g) and $\mathrm{NaBr}$ sodium bromide ( $0.3 \mathrm{~g}$ ) solids and bleach (37.22 g, $6 \mathrm{wt} \% \mathrm{NaOCl})$ to as-received cellulose ( $3 \mathrm{~g}$ ) under vigorous stirring. $10 \mathrm{~mL}$ of $0.5 \mathrm{M} \mathrm{NaOH}$ solution were added after $1 \mathrm{hr}$, followed by another $10 \mathrm{~mL}$ of the $0.5 \mathrm{M} \mathrm{NaOH}$ solution at 2 $\mathrm{hr}$. Finally, after $5 \mathrm{hr}, 15 \mathrm{~mL}$ of ethanol were added to quench the reaction. The oxidized cellulose was then centrifuged and rinsed with DI water three times before refrigerating and exchanging the water with DMF. PVDF films with added water (series C) and cellulose nanofibril/PVDF films obtained from distilled reagent grade DMF (Sigma Aldrich 319937) and as-received cellulose slurry were also obtained (series D). The as-received, aqueous, cellulose slurry could not be directly incorporated into PVDF dissolved with anhydrous DMF. A summary of the films series pertaining to the cellulose nanofibril/PVDF study, and their processing conditions, are listed in Table 2. Appendix D shows preliminary FTIR measurements obtained from cellulose nanofibril/PVDF films obtained using high temperature melting. Under those processing conditions, $\alpha$ to $\gamma$ phase transformation is observed.

\subsection{Characterization techniques}

The surface morphology of the films was probed with polarized optical microscopy (Zeiss) under variable optical magnification. SEM imaging was also carried out on the constituents and the films with a FEI Nova Nano-SEM 630 microscope equipped with a secondary electron detector. For SEM imaging, the accelerating voltages were set between 5 $\mathrm{kV}$ and $10 \mathrm{kV}$ and the spot size between 2.0 and 5.0. In order to reduce 
electron beam-induced surface charging, samples were coated with a thin layer of gold prior to SEM imaging.

Table 2. Summary of experimental processing conditions and films series studied in this report.

\begin{tabular}{|l|l|}
\hline Sample ID & \multicolumn{1}{c|}{ Composition/Processing conditions } \\
\hline Series A & $\begin{array}{l}\text { Anhydrous DMF, as-received cellulose slurry incorporated after } \\
\text { solvent exchange, } \mathrm{T}=100^{\circ} \mathrm{C} \text { drying overnight in oven. }\end{array}$ \\
\hline Series B & $\begin{array}{l}\text { Anhydrous DMF, TEMPO-mediated oxidized cellulose slurry } \\
\text { incorporated after solvent exchange, } \mathrm{T}=100^{\circ} \mathrm{C} \text { drying overnight in } \\
\text { oven. }\end{array}$ \\
\hline Series C & $\begin{array}{l}\text { Anhydrous DMF, water added to PVDF/DMF solution, } \mathrm{T}=100^{\circ} \mathrm{C} \\
\text { drying overnight in oven (Appendix A). }\end{array}$ \\
\hline Series D & $\begin{array}{l}\text { Distilled reagent grade DMF, as-received cellulose slurry } \\
\text { incorporated (no solvent exchange), } \mathrm{T}=100^{\circ} \mathrm{C} \text { drying overnight in } \\
\text { oven (Appendix C). }\end{array}$ \\
\hline
\end{tabular}

The cellulose nanofibril slurry was further characterized with a Bruker Dimension Icon (Bruker) Atomic Force Microscope. The slurry was dispersed onto freshly cleaved mica substrates following the procedure outlined by Sacui et al. (2014). Briefly, the substrates were prepared by drop casting PLL solution (0.01\% by mass) onto the mica substrates (Ted Pella 50), which were blown dry after $5 \mathrm{~min}$. Diluted cellulose suspensions ( 0.02\%-0.03\% by mass) were drop-cast onto the PLLcoated mica substrates. After $5 \mathrm{~min}$, the substrates were gently rinsed with DI water to remove unattached nanocellulose and blown dry. AFM topographical images were captured using the ScanAsyst mode using a tip with a 2-nm nominal tip radius. AFM data were plotted and processed with Gwyddion (Necas and Klapetek 2012).

X-ray diffraction was performed on the composite films to identify variations in the blended materials using a Panalytical X'Pert Pro materials diffractometer equipped with a Cobalt-Ka X-ray source. $\theta-2 \theta$ diffraction patterns were collected from 2 to $70^{\circ}$ with a step size of 0.02. FTIR spectra between 700 and $4000 \mathrm{~cm}^{-1}$ were collected from dried cellulose (as-received and oxidized), neat PVDF, and cellulose nanofibril/PVDF films with a Nicolet 6700 FTIR spectrometer equipped with an attenuated total reflectance (ATR) accessory. The area probed by the IR beam was approximately $1.5 \mathrm{~mm}$ in diameter, and three spectra were collected per sample. The obtained FTIR spectra were leveled by subtracting a linear background spanning the entire spectral 
range. Since the intensity of the $1070 \mathrm{~cm}^{-1}$ band has a linear dependence on film thickness and is independent of the polymer crystalline phases, the FTIR spectra of all films were normalized with respect to the $1070 \mathrm{~cm}^{-1}$ peak (Martins et al. 2014).

The melting behavior of the composites was investigated with a differential scanning calorimeter (DSC 250, TA instruments). A heat-cool-heat cycle was employed from -50 to $250^{\circ} \mathrm{C}$ at $5^{\circ} \mathrm{C} / \mathrm{min}$ heating and cooling rates. Peak maximum of the melting endotherm from the $1^{\text {st }}$ heating segment was recorded and referred to as $T_{m}$. Melting endotherms were integrated and used to determine percent crystallinity based upon 104.5 J/g for the 100\% crystalline material (Wang and Chen 2016).

Tensile (stress versus strain) properties of the films were evaluated with a hybrid rheometer (TA instruments, Discovery) functioning as a dynamic mechanical analyzer in tension mode. Samples were cut into $\sim 5$ $\times 15 \mathrm{~mm}$ strips along the draw direction, and tests were conducted at a constant linear rate of $10 \mu \mathrm{m} / \mathrm{s}$. For dynamic assays to determine elastic modulus, data were acquired from 25 to $45^{\circ} \mathrm{C}$ with a heating rate of $5^{\circ} \mathrm{C} / \mathrm{min}$, a strain of $0.1 \%$, and the frequency value of $1 \mathrm{~Hz}$. 


\section{Results}

SEM imaging of the as-received cellulose slurry confirmed the presence of high-aspect ratio cellulose nanofibrils (Figure $3[\mathrm{~A}]$ ), whereas higher resolution AFM imaging also revealed the presence of finer particles, which are likely cellulose nanocrystals (Figure $3[\mathrm{~B}]$ ). Cellulose nanofibrils are long, high-aspect ratio fibers consisting of long chains of alternating crystalline and amorphous cellulose. Acid hydrolysis of the nanofibrils breaks them down into crystalline cellulose nanocrystals. The surface charge of the cellulose nanofibrils is neutral ( $\mathrm{OH}$ terminated), whereas cellulose nanocrystals, typically obtained from sulfuric acid hydrolysis, have negative surface chemistry $\left(\mathrm{OSO}_{3}{ }^{-}\right.$termination). The PLL-coated mica substrates are positively charged, thereby favoring the attachment of negatively charged particles such as cellulose nanocrystals. According to the manufacturer specifications, the nanofibril slurry contains 3-4 wt\% solids with the majority of the solids in the form of nanofibrils. Figure $3[\mathrm{C}]$ shows the morphology of the oxidized cellulose obtained after the TEMPOmediated oxidation treatment, revealing the presence of nanofibrils that are, on average, finer than the ones in the as-received cellulose slurry.

Figure 3. Characterization of cellulose. A. SEM image showing high-aspect ratio fibrils of the as-received cellulose. B. AFM scan revealing cellulose nanocrystals, in addition to cellulose nanofibrils. C. AFM of TEMPO-mediated oxidized cellulose revealing the presence of finer particles after the TEMPO treatment. AFM scan sizes are $5 \mu \mathrm{m} \times 5$ $\mu \mathrm{m}$.
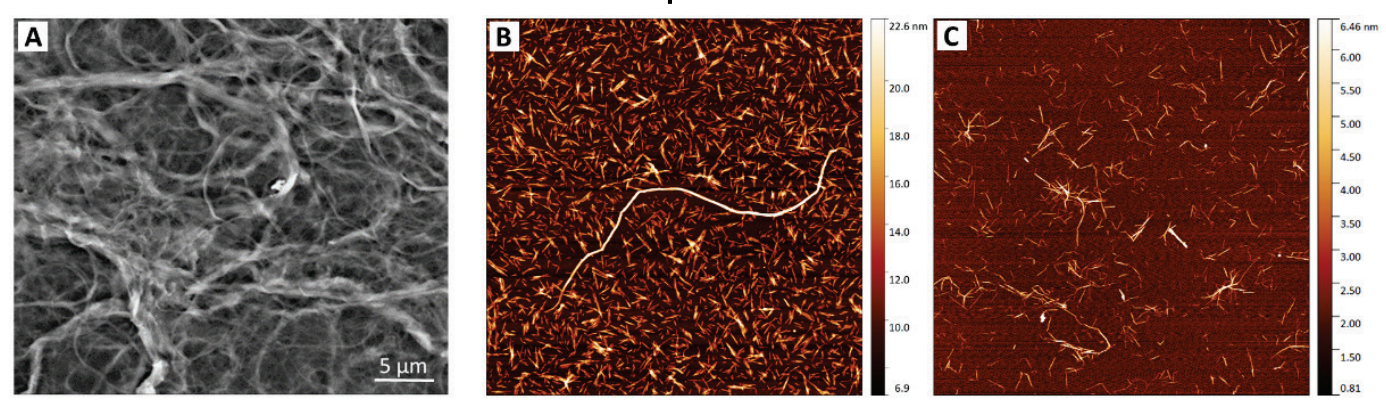

The FTIR spectra, from 600 to $3800 \mathrm{~cm}^{-1}$, of dried, as-received, and TEMPO-mediated oxidized cellulose are shown in Figure 4. The spectra for the as-received and oxidized cellulose share many characteristic peaks such as the broad peak between $3000-3650 \mathrm{~cm}^{-1}$ due to $-\mathrm{OH}$ stretching, $-\mathrm{CH}$ stretching at $2897 \mathrm{~cm}^{-1}$, and $-\mathrm{CH}_{2}$ wagging at $1315 \mathrm{~cm}^{-1}$ (Han et al. 2013). For the as-received cellulose, the broad peak around $1645 \mathrm{~cm}^{-1}$ corresponds to the $\mathrm{OH}$ bending mode of adsorbed water. The peak at $811 \mathrm{~cm}^{-1}$ has been attributed to the symmetrical -COS vibration of the $\mathrm{COSO}_{3}$ functional group 
due to esterification of hydroxyl groups by sulfate ions, which occurs during the acid hydrolysis treatment of cellulose (Han et al. 2013). The spectrum of TEMPO-mediated oxidized cellulose shows peaks characteristic of the oxidation process at $1602 \mathrm{~cm}^{-1}$ and $1409 \mathrm{~cm}^{-1}$ corresponding to surface carboxylate groups (-COO- $\mathrm{Na}^{+}$, -COO-) (Cheng et al. 2016).

Figure 4. FTIR spectra of as-received and TEMPO-mediated oxidized cellulose.

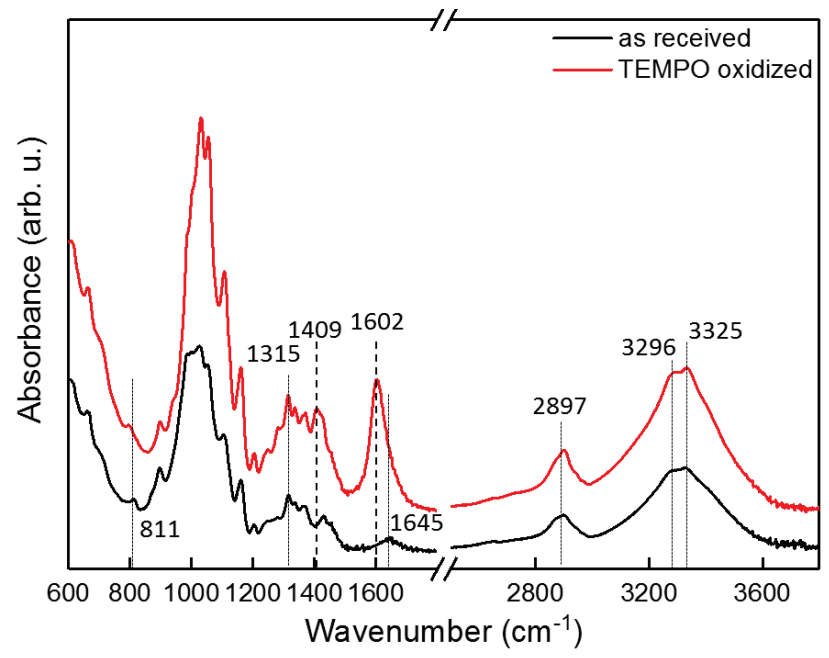

The surface morphology of the as-received and oxidized cellulose nanofibril/PVDF films is shown in Figure 5 and Figure 6, respectively. The neat PVDF film (Figure 5 [A]) has a relative smooth surface consisting of densely interconnected spherulites. For films loaded with as-received cellulose nanofibrils, both the porosity and surface roughness increase with increasing loading. Under higher loadings, cellulose fibrils partially protrude from the film surface (for example see Figure $5[\mathrm{~F}]-$ $5[G])$. The surface morphology of films obtained with oxidized cellulose is shown in Figure 6. Under low loading (Figure 6[A]-[B]), the films exhibit a combination of smooth and rough (pockmarked) areas. As the cellulose concentration increases, the surface roughness increases with large craters appearing throughout the film surface.

X-ray diffraction was carried out to determine the crystalline structure of the composites' films, and their diffraction patterns are shown in Figure 7. In general, all diffraction patterns show two prominent peaks at approximately $21.6^{\circ}$ and $23.6^{\circ}$, which correspond to the (020) and (110) reflections of $\gamma$ PVDF (Martins et al. 2014). The smaller peak at $\sim 46^{\circ}$ is also assigned to the (210) $\gamma$ reflection. Phase information of the films can be obtained also from FTIR spectroscopy. The FTIR spectra of as-received cellulose nanofibrils, neat PVDF, and as-received cellulose 
nanofibril/PVDF composites are shown in Figure 8[A]. The FTIR spectrum of the cellulose nanofibrils is the same as the one shown in Figure 4. As indicated by the absorption bands at $811 \mathrm{~cm}^{-1}$ and $1232 \mathrm{~cm}^{-1}$, and in good agreement with X-ray diffraction, the neat PVDF film crystallizes predominantly in the $\gamma$ phase (Martins et al. 2014). The relative crystalline fraction of the $\gamma$ phase $(F(\gamma))$ can be calculated from:

$$
F(\gamma)=\frac{X_{\gamma}}{X_{\gamma}+X_{\alpha}}=\frac{A_{\gamma}}{\left(K_{\gamma} / K_{\alpha}\right) A_{\alpha}+A_{\gamma}}
$$

where:

$$
\begin{aligned}
F(\gamma) & =\text { relative amount of } \gamma \text { phase } \\
X_{\gamma} & =\text { degree of crystallinity of } \gamma \text { phase } \\
X_{\alpha} & =\text { degree of crystallinity of } \alpha \text { phase } \\
K_{\gamma} & =\text { absorption coefficient of } \gamma \text { phase }\left(0.15 \mu \mathrm{m}^{-1}\right) \\
K_{\alpha} & =\text { absorption coefficient of } \alpha \text { phase }\left(0.365 \mu \mathrm{m}^{-1}\right) \\
A_{\gamma} & =\text { FTIR absorbance at } 833 \mathrm{~cm}^{-1} \\
A_{\alpha} & =\text { FTIR absorbance at } 764 \mathrm{~cm}^{-1}
\end{aligned}
$$


Figure 5. SEM images of the film surface for A. Neat PVDF, B. 0.5 wt\%, C. 1 wt\%, D. 2 wt\%, E. 3 wt\%, F. 4 wt\% and G. 5 wt\% cellulose nanofibril/PVDF composites (series A).
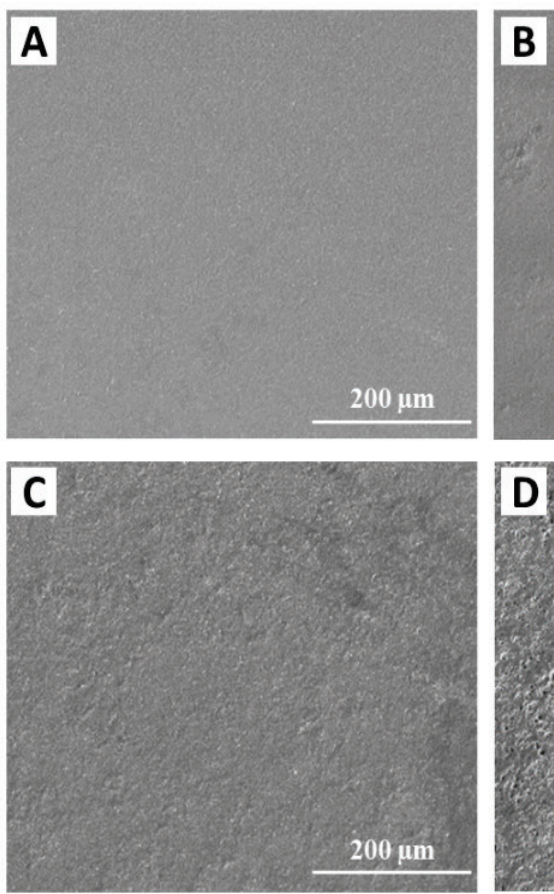

$200 \mu \mathrm{m}$
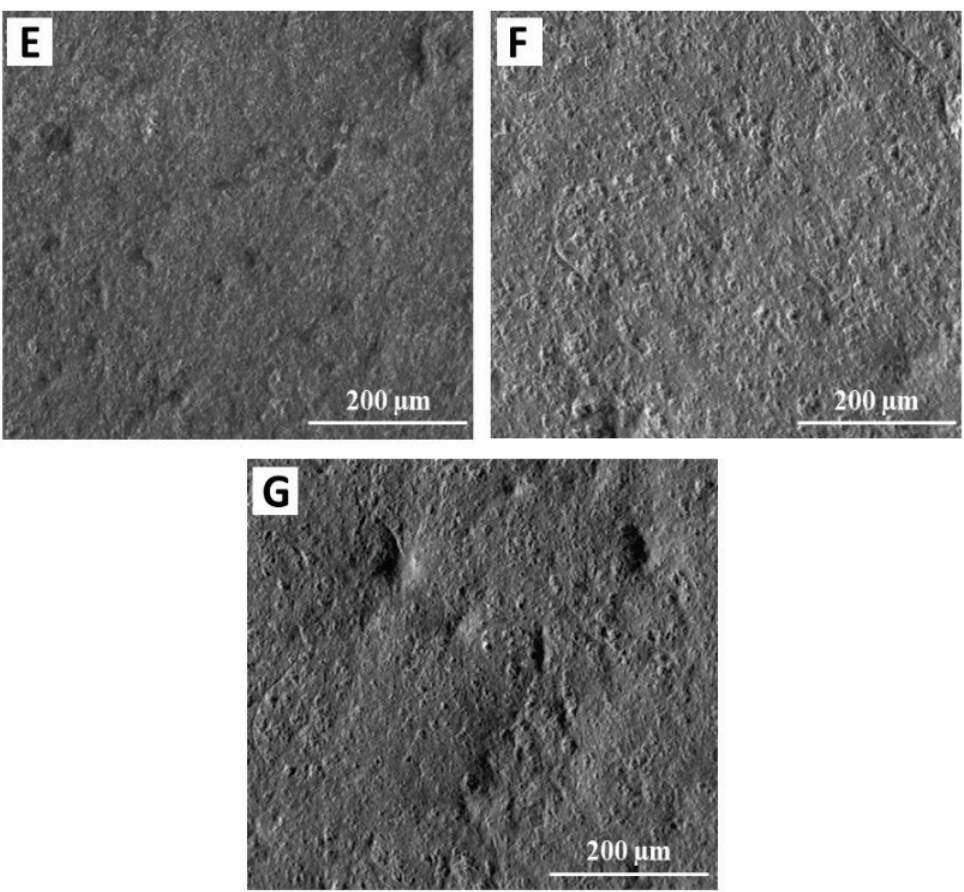
Figure 6. SEM images of the film surface for A. $0.5 w t \%$, B. 1 wt\%, C. 2 wt\%, D. 3 wt\%, E. 4 wt\% and F. 5 wt\% oxidized cellulose nanofibril/PVDF composites (series B).
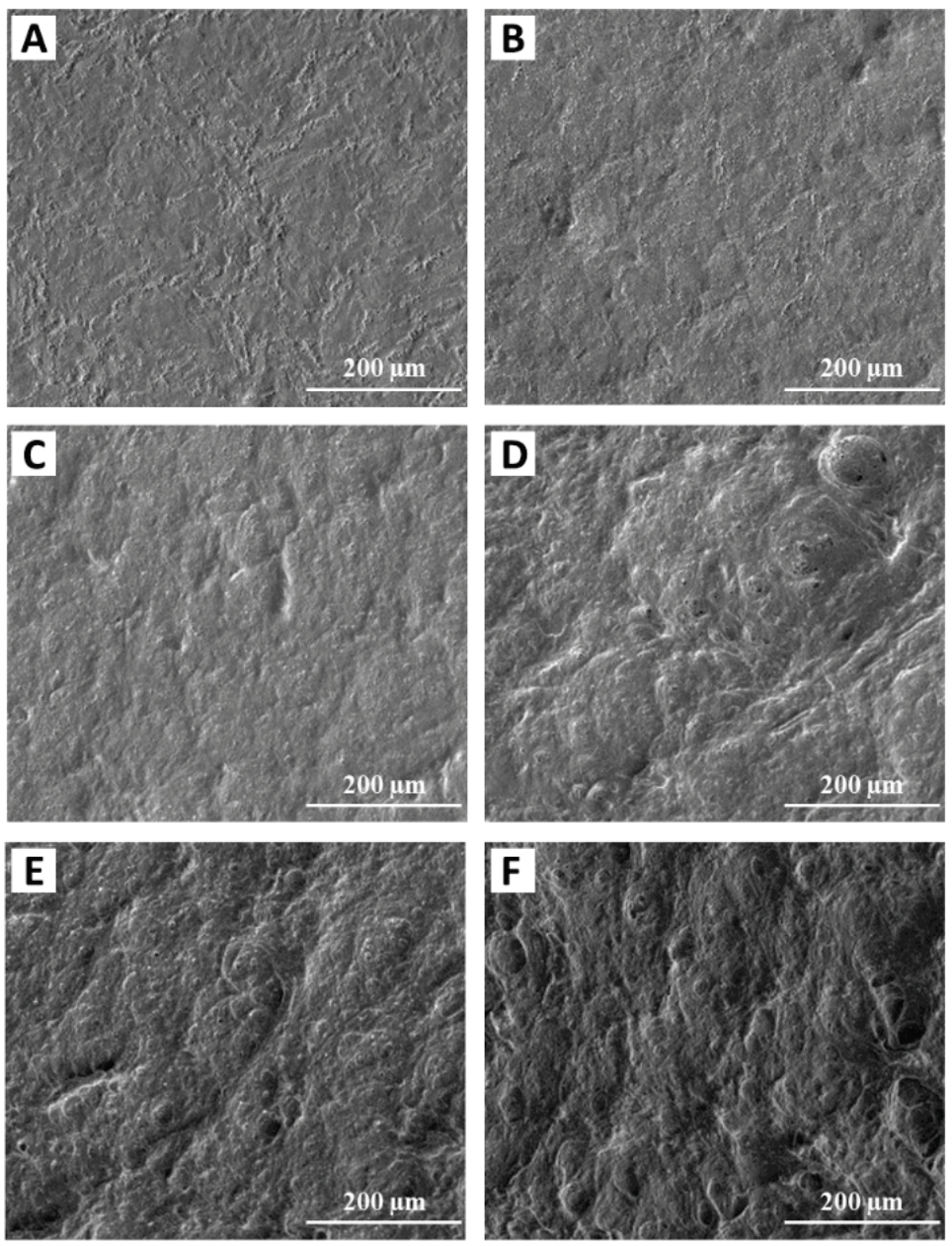
Figure 7. XRD patterns for A. As-received cellulose nanofibril/PVDF composites (series A); and B. Oxidized cellulose nanofibril/PVDF composites (series B).
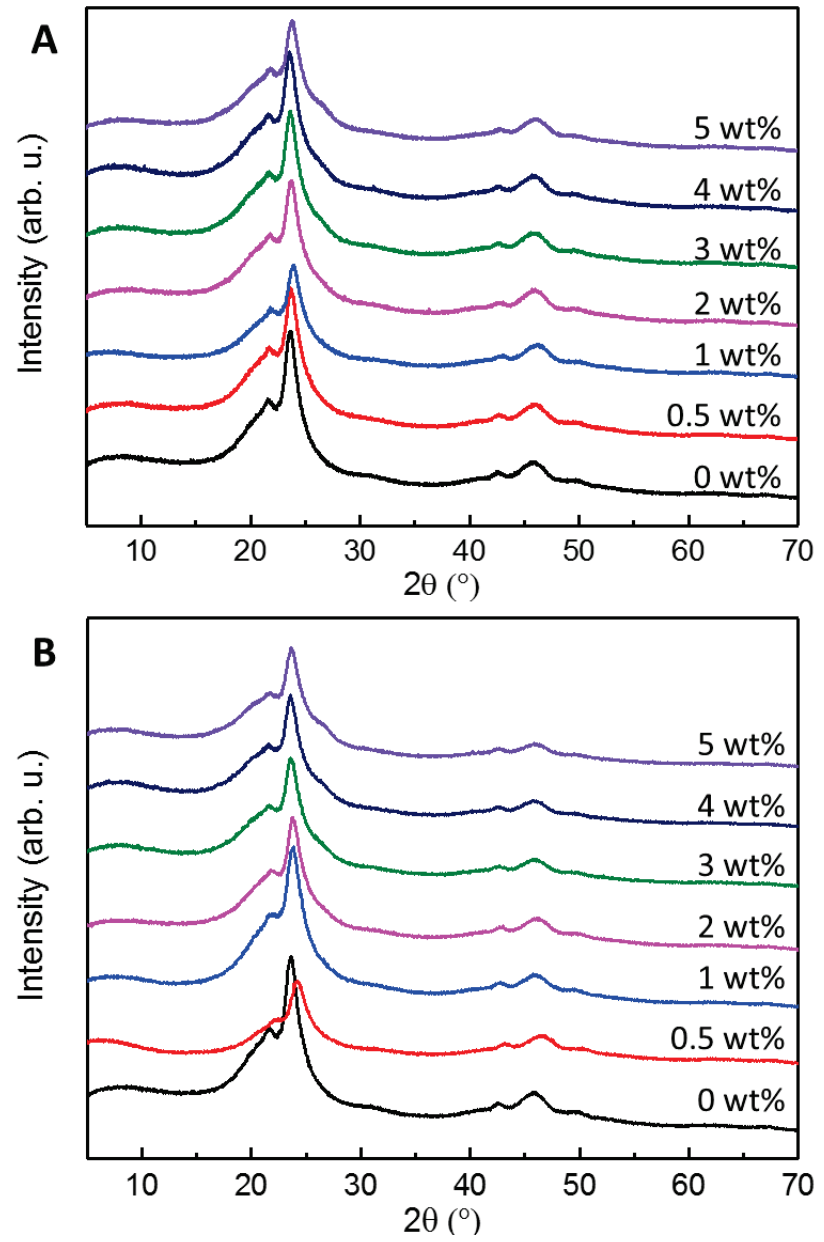

$F(\gamma)$ was calculated to be approximately $89.5 \%$ for the neat film, whereas for the composite films $F(\gamma)$ slightly increases with increasing loading (refer to Table 3). Qualitative information regarding the $\alpha$ and $\gamma$ phases can be obtained by calculating the FTIR absorbance intensity ratios $\mathrm{A}_{764} / \mathrm{A}_{874}$ and $\mathrm{A}_{1232} / \mathrm{A}_{874}$, where $\mathrm{A}_{764}, \mathrm{~A}_{874}$, and $\mathrm{A}_{1232}$ are the absorbances at $764 \mathrm{~cm}^{-1}, 874 \mathrm{~cm}^{-1}$, and $1232 \mathrm{~cm}^{-1}$, respectively (Imran-ul-haq et al. 2008). The intensity ratios $A_{1275} / A_{874}$ and $A_{840} / A_{874}$, involving peaks related to the $\beta$ phase, were also calculated, but they are of little interest due to the lack of distinctive $\beta$ phase peaks in the films. Figure 8 [B] shows the aforementioned intensity ratios for the as-received cellulose nanofibril/PVDF series. $\mathrm{A}_{1232} / \mathrm{A}_{874}$ gradually increases and plateaus at 2 wt\% loading, indicating an increase in the $\gamma$ phase content, whereas the $\mathrm{A}_{764} / \mathrm{A}_{874}$ ratio decreases indicating a decrease in $\alpha$ phase. The FTIR spectra and the relevant FTIR peak intensity ratios for the oxidized cellulose nanofibril/PVDF films are shown in Figure 9[A]-[B]. $\mathrm{F}(\gamma)$ for 
the oxidized cellulose nanofibril/PVDF is also listed in Table 3, with the highest amount of $\mathrm{F}(\gamma)(\sim 92 \%)$ observed for 1 to $3 \mathrm{wt} \%$ loading. Overall, $\mathrm{A}_{1232} / \mathrm{A}_{874}$ increases up to $1 \mathrm{wt} \%$ and then plateaus for higher cellulose loadings. To the detection limit of the research team's instrument, no hydroxyl peaks were observed in the $3100-3600 \mathrm{~cm}^{-1}$ region for either set of films (see Figure 10) indicating the absence of measurable hydrogen bonding between the dopants with the PVDF lattice.

Figure 8. A. FTIR spectra of neat PVDF and as-received cellulose nanofibril/PVDF composites (series A). B. Calculated intensity ratios for qualitative estimation of $\alpha, \beta$, and $\gamma$ phases in the composite films as function of cellulose wt\%.
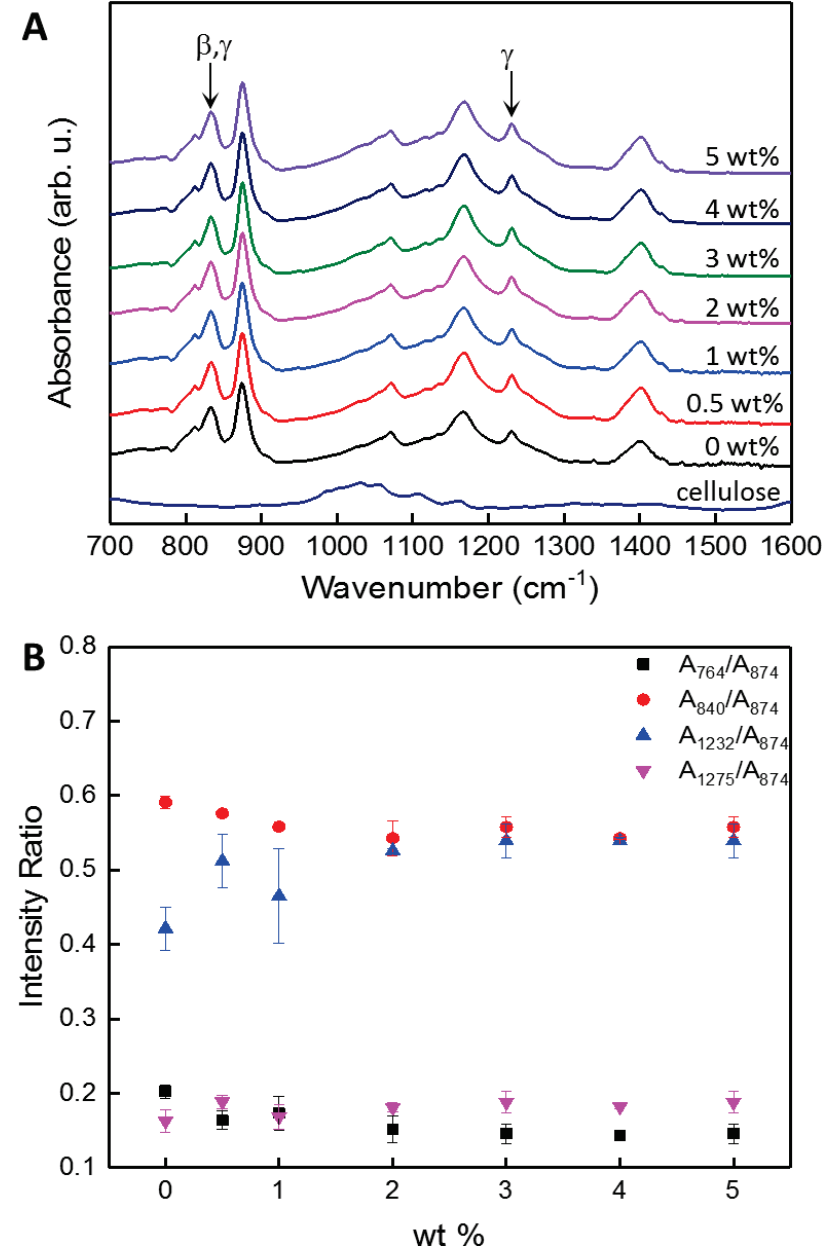

The melting behavior of the nanocomposites was evaluated with DSC in a heat-cool cycle with relevant parameters such as the heat of fusion $\left(\Delta H_{f}\right)$, peak melting temperature $\left(T_{m}\right)$, and percent crystallinity $\left(X_{c}\right)$ listed in Table 3. As shown in Figure 11, incorporation of as-received (series A) or oxidized nanocellulose (series B) shifts the melting peak to lower temperatures suggesting a strong interaction between cellulose nanofibrils and PVDF. In 
addition, the melting points of composites with oxidized cellulose nanofibrils are also lower than those of as-received cellulose. For PVDF, it has been reported that the melting temperatures for the $\alpha$ and $\beta$ phases are in the $167-172^{\circ} \mathrm{C}$ range, and the $\gamma$ phase in the $179-180^{\circ} \mathrm{C}$ when obtained by crystallization from the melt, and even higher from $\alpha$ to $\gamma$ transformation (Martins et al. 2014). However, the melting behavior of the composites is dependent not only on the crystalline phases in the films, but also by the presence of defects, which are affected both by the processing conditions and the type of additive. Similarly, the molecular weight of PVDF also affects the melting temperatures (Issa et al. 2016). Two factors are likely contributing to the reduction of the melting temperature: first, reduction in the polymer spherulite size, as observed with optical microscopy; and second, interactions between the surface functional groups of nanocellulose and the PVDF matrix. Interestingly, the crystallinity of the as-received cellulose nanofibril/PVDF composites (with the 3 wt\% composite being an exception) is higher than that of neat PVDF and also higher than that of the oxidized cellulose nanofibril PVDF composites.

Table 3. FTIR crystalline phase fraction and DSC results for as-received cellulose nanofibril/PVDF films (series A) and oxidized cellulose nanofibril/PVDF films (series B).

\begin{tabular}{|l|l|l|l|l|l|}
\hline & \multicolumn{1}{|c|}{ wt\% } & \multicolumn{1}{c|}{$\mathrm{F}(\gamma)$} & \multicolumn{1}{c|}{$\Delta H_{f}(\mathrm{~J} / \mathrm{g})$} & \multicolumn{1}{c|}{$\boldsymbol{X}_{c}(\%)$} & \multicolumn{1}{c|}{$\boldsymbol{T}_{m}$} \\
\hline \multirow{5}{*}{ Series A } & 0 & 0.89 & 52.1 & 49.8 & 172.2 \\
\cline { 2 - 6 } & 0.5 & 0.91 & 54.7 & 52.6 & 170.6 \\
\cline { 2 - 6 } & 1 & 0.91 & 55.0 & 53.1 & 170.8 \\
\cline { 2 - 6 } & 2 & 0.91 & 52.8 & 51.5 & 170.1 \\
\cline { 2 - 6 } & 3 & 0.91 & 48.2 & 47.5 & 170.4 \\
\cline { 2 - 6 } & 4 & 0.92 & 50.4 & 50.2 & 170.9 \\
\cline { 2 - 6 } & 5 & 0.92 & 54.3 & 54.6 & 170.5 \\
\hline \multirow{5}{*}{ Series B } & 0.5 & 0.91 & 51.0 & 49.0 & 169.8 \\
\cline { 2 - 6 } & 1 & 0.92 & 50.7 & 49.0 & 169.9 \\
\cline { 2 - 6 } & 2 & 0.92 & 48.6 & 47.4 & 168.9 \\
\cline { 2 - 6 } & 3 & 0.92 & 49.5 & 48.8 & 168.6 \\
\cline { 2 - 6 } & 4 & 0.91 & 46.2 & 46.0 & 169.4 \\
\cline { 2 - 6 } & 5 & 0.91 & 48.7 & 49.0 & 169.4 \\
\hline
\end{tabular}

The mechanical properties (tensile strength, tensile modulus, \% elongation at break, yield strength, and tensile toughness) of the films are listed in Table 4. The tensile strength of the as-received cellulose nanofibril/PVDF composites is reduced by approximately $20 \%$ compared to the neat film, but does not 
have a strong dependence with the wt\% of the cellulose. On the other hand, the modulus shows a minimum for the $2 \mathrm{wt} \%$ film and then increases, roughly reaching that of the neat film for $4 \mathrm{wt} \%$. As evidenced by the reduction in the \% elongation at break, the incorporation of cellulose reduces the ductility of the composites. The oxidized cellulose nanofibril/PVDF films exhibit a much stronger dependence of mechanical properties on the amount of oxidized cellulose present in the films. In particular, up to $4 \mathrm{wt} \%$, the tensile strength decreases linearly with increasing oxidized cellulose nanofibril wt\% and plateaus to a minimum value of $\sim 15 \mathrm{MPa}$. The modulus of the oxidized cellulose nanofibril/PVDF series shows an increase for the 1 wt\% film, but decreases for higher loadings. Similar to the as-received cellulose nanofibril/PVDF films, the \% elongation at break for the oxidized cellulose nanofibril/PVDF series decreases with increasing loading. The yield stress of the cellulose nanofibril/PVDF films remains relatively constant regardless of added cellulose, but the tensile toughness decreases within increasing loading. The oxidized cellulose/PVDF composites show a reduction of both yield stress and tensile toughness with increasing loading. The differences in the mechanical behavior of the as-received and oxidized cellulose nanofibrils/PVDF can be attributed to the morphological changes of cellulose after the oxidation treatment as well as effects of surface functionalization. The oxidized cellulose is generally finer than the asreceived cellulose and has a higher amorphous content; therefore, it is not as mechanically robust as the as-received cellulose. Also, TEMPO-mediated oxidized cellulose does not form stable dispersions in DMF (Okita et al. 2011). The lack of cellulose dispensability can cause fibril agglomeration, which can lead to reduction of mechanical strength in the composites' films. 
Figure 9. A. FTIR spectra of neat PVDF and oxidized cellulose nanofibril/PVDF composites (series B). B. Calculated intensity ratios for qualitative estimation of $\alpha, \beta$, and $\gamma$ phases in the composite films as function of oxidized cellulose nanofibril wt\%.
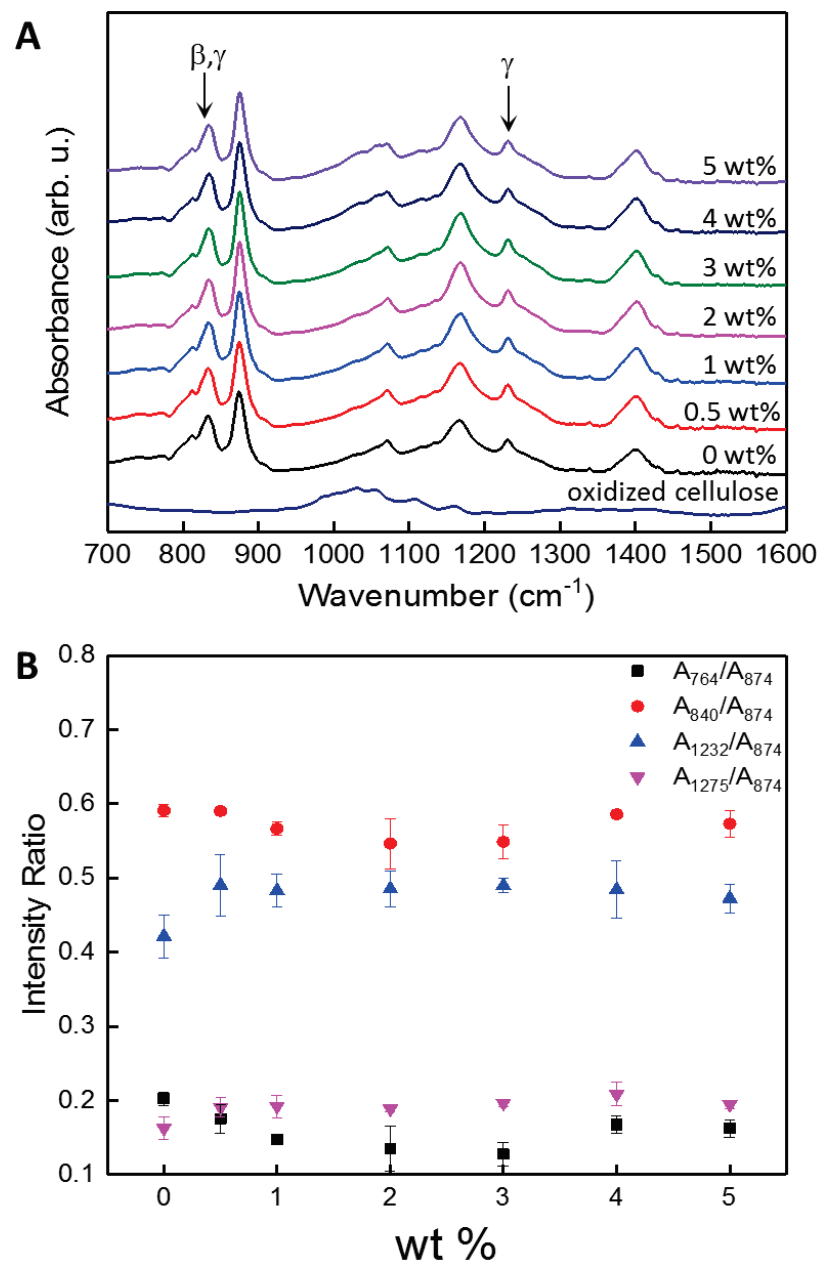
Figure 10. FTIR spectra in the $3100-3600 \mathrm{~cm}^{-1}$ range for $A$. as-received (series $A$ ), and B. oxidized cellulose nanofibril/PVDF composites (series B). No hydroxyl peaks were observed for either film series.
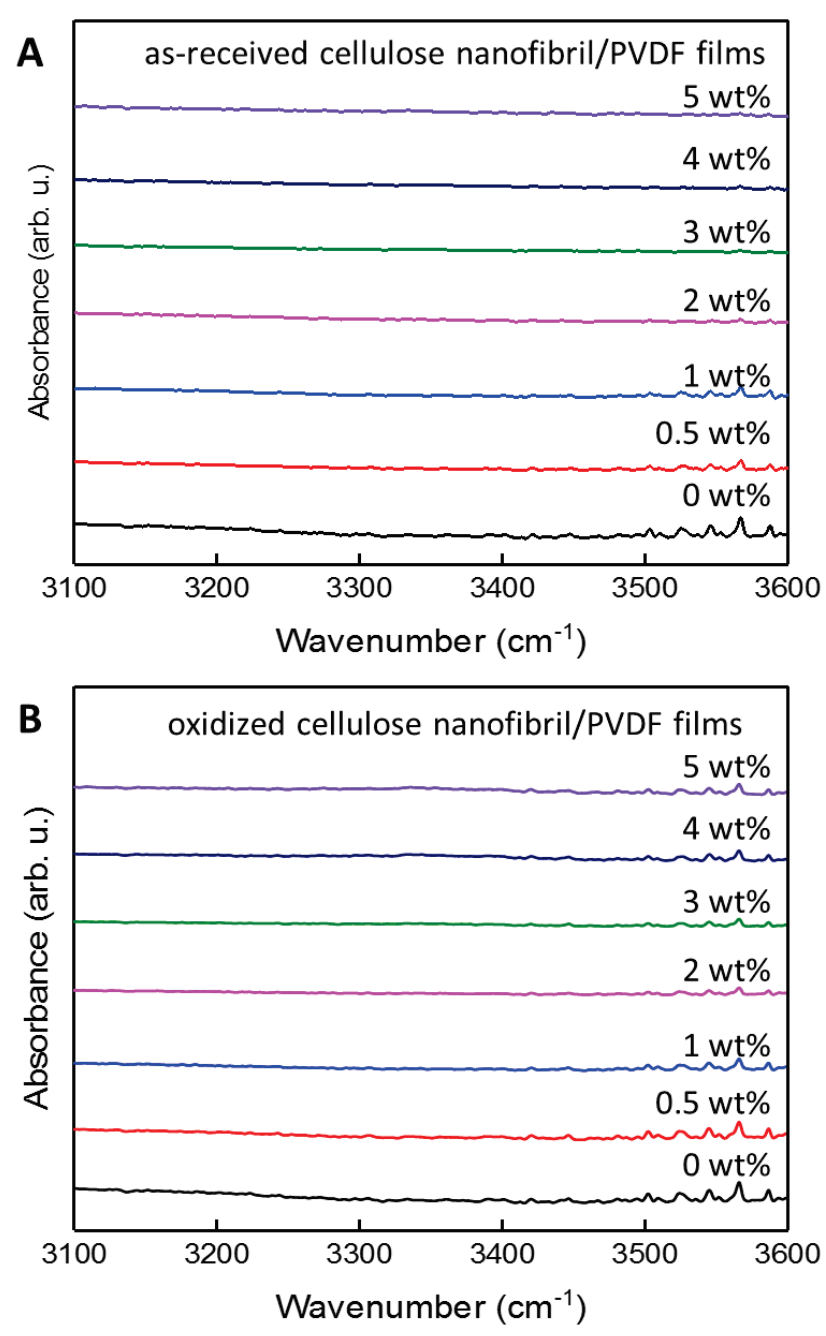
Table 4. Tensile properties of as-received cellulose nanofibril/PVDF films (Series A) and oxidized cellulose nanofibril/PVDF films (Series B).

\begin{tabular}{|c|c|c|c|c|c|c|}
\hline & wt\% & $\begin{array}{c}\text { Tensile } \\
\text { strength } \\
\text { (MPa) }\end{array}$ & $\begin{array}{c}\text { Tensile } \\
\text { Modulus } \\
(\mathrm{MPa})\end{array}$ & $\begin{array}{l}\text { Elong. at } \\
\text { break (\%) }\end{array}$ & $\begin{array}{l}\text { Yield } \\
\text { stress } \\
(\mathrm{MPa}) \\
\end{array}$ & $\begin{array}{c}\text { Tensile } \\
\text { toughness } \\
\left(10^{3}\right. \\
\left.\mathrm{kJ} / \mathrm{m}^{3}\right)\end{array}$ \\
\hline \multirow{7}{*}{ Series A } & 0 & $54.7 \pm 5.9$ & $1121 \pm 29$ & $37.7 \pm 4.2$ & $29.2 \pm 1.4$ & $17.6 \pm 3.1$ \\
\hline & 0.5 & $43.4 \pm 6.8$ & $1215 \pm 184$ & $14.1 \pm 1.1$ & $21.1 \pm 3.2$ & $4.5 \pm 1.1$ \\
\hline & 1 & $43.5 \pm 9.5$ & $1081 \pm 282$ & $11.7 \pm 2.9$ & $20.4 \pm 1.7$ & $3.6 \pm 1.5$ \\
\hline & 2 & $41.1 \pm 0.5$ & $747 \pm 38$ & $19 \pm 7.6$ & $21.7 \pm 1.9$ & $6.5 \pm 3.0$ \\
\hline & 3 & $45.6 \pm 1.4$ & $944 \pm 39$ & $10.2 \pm 0.0$ & $21.6 \pm 1.2$ & $3.4 \pm 0.1$ \\
\hline & 4 & $47.1 \pm 3.9$ & $1090 \pm 10$ & $10.8 \pm 0.3$ & $25.1 \pm 0.4$ & $3.7 \pm 0.3$ \\
\hline & 5 & $42.5 \pm 1.6$ & $1053 \pm 77$ & $11.7 \pm 2.1$ & $21.5 \pm 4.8$ & $3.4 \pm 0.7$ \\
\hline \multirow{6}{*}{ Series B } & 0.5 & $47.8 \pm 5.4$ & $1134 \pm 56$ & $18.1 \pm 5.7$ & $21.1 \pm 3.1$ & $6.6 \pm 2.5$ \\
\hline & 1 & $41.5 \pm 4.9$ & $1331 \pm 48$ & $12.2 \pm 0.9$ & $23.8 \pm 2.6$ & $3.8 \pm 0.7$ \\
\hline & 2 & $33.3 \pm 0.6$ & $747 \pm 6$ & $8.1 \pm 0.3$ & $20.7 \pm 0.6$ & $1.8 \pm 0.1$ \\
\hline & 3 & $24.6 \pm 3.8$ & $647 \pm 121$ & $6.6 \pm 0.6$ & $17.9 \pm 1.2$ & $1.0 \pm 0.2$ \\
\hline & 4 & $15.8 \pm 1.8$ & $196 \pm 20$ & $5.1 \pm 0.6$ & $11.4 \pm 1.1$ & $0.4 \pm 0.1$ \\
\hline & 5 & $14.6 \pm 2.7$ & $477 \pm 38$ & $4.6 \pm 0.9$ & $11.4 \pm 0.7$ & $0.3 \pm 0.2$ \\
\hline
\end{tabular}


Figure 11. DSC showing the melting behavior of the neat PVDF, and A. as-received (series A), B. oxidized cellulose nanofibril/PVDF composites (series B).
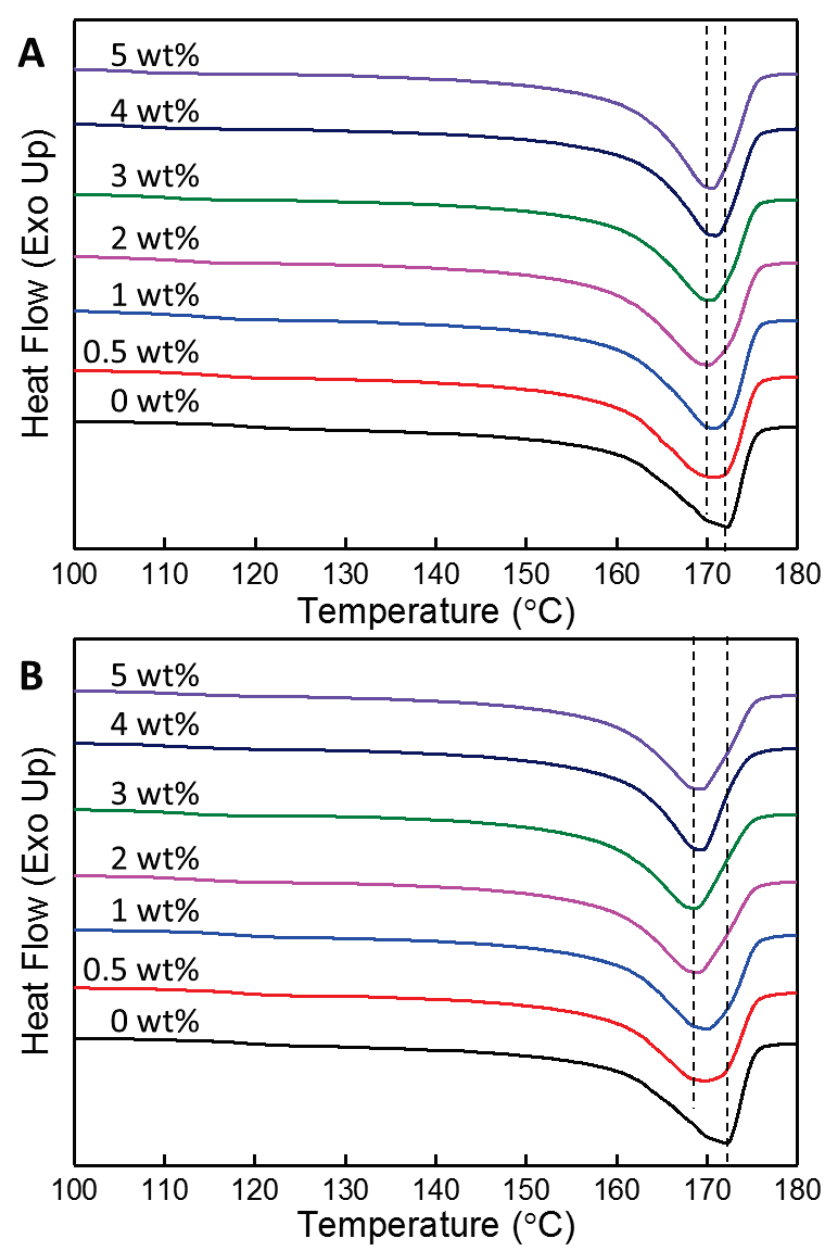


\section{Conclusions and Recommendations}

The physico-mechanical properties of PVDF can be altered through incorporation of cellulose nanofibrils. Under the current processing conditions, cellulose nanofibrils have a negligible effect on inducing crystalline phase change in the composites, but do affect certain physico-mechanical properties of the composites (e.g., melting behavior, tensile mechanical properties, etc.) The findings of this study can be summarized as follows:

- Composite films obtained from solvent evaporation at $100^{\circ} \mathrm{C}$ with solvent exchanged cellulose nanofibrils, remain in a majority polar phase $(\gamma)$ regardless of the amount of added cellulose nanofibrils.

- Addition of as-received cellulose nanofibrils reduces the tensile strength and modulus of the composites, whereas incorporation of TEMPO-mediated oxidized cellulose nanofibrils has a pronounced negative effect on these properties. The yield stress of the as-received cellulose nanofibril/PVDF films remains approximately constant, but the tensile toughness is gradually reduced.

- Composite films obtained from solvent evaporation at $100^{\circ} \mathrm{C}$ with asreceived aqueous cellulose slurry, exhibit nucleation of minority $\alpha$ and $\beta$ phases (see Appendix B). It is hypothesized that hydrated cellulose nanofibrils retain water during the solvent evaporation process, leading to prolonged interaction of water molecules with the PVDF lattice and thereby inducing local PVDF chain changes.

- For the employed processing conditions, cellulose nanofibrils do not induce $\gamma$ to $\beta$ phase transformation, as initially hypothesized.

- Similarly, for the employed processing conditions, $1.5 \mathrm{wt} \%$ DNA/PVDF films do not show $\gamma$ to $\beta$ phase transformation (see Appendix D).

This investigation was a preliminary study on the effect of cellulose on various cellulose/PVDF composite properties. From the prospective of Army applications, the composite cellulose/PVDF films are not ideal because their tensile properties are reduced with the addition of cellulose. The reduction in these properties can be attributed to the fundamental incompatibility between hydrophilic cellulose and hydrophobic PVDF. Recommendations for future work include refinement of the film processing conditions. More specifically, improved dispersion of cellulose in solvents such as DFM or DMSO and minimization of water content in each step of the film fabrication process and are key processing conditions 
that need to be improved. First, functionalization of cellulose with $-\mathrm{COOH}$ instead of -COONa will enable its dispersion in DMF, allowing improved dispersion in the dissolved PVDF. Second, drying the composite films in a vacuum oven will aid in reducing water infiltration in the composite solutions and minimize vapor-induced phase separation, which will in turn yield films with increased morphological homogeneity.

Regarding the potential of cellulose to induce a crystalline phase transformation in PDVDF, conversion from $\alpha$ to $\gamma$ PVDF and not $\gamma$ to $\beta$ PVDF is most commonly reported in literature for nanoparticle/PVDF composites. Therefore, solvent evaporation at higher temperature (> $120^{\circ} \mathrm{C}$ ) or melting is recommended in order to obtain neat $\alpha$ phase PVDF. Encouraging preliminary results on the effect of cellulose on $\alpha$ phase PVDF are shown in Appendix D; namely, with increasing cellulose loading, how composite films increased $\gamma$ phase content. 


\section{References}

Bar-Cohen, Y., and Q. Zhang. 2008. Electroactive polymer actuators and sensors. MRS Bulletin 33:173-181.

Beeby, S. P., M. J. Tudor, and N. M. White. 2006. Energy harvesting vibration sources for microsystems applications. Measurement Science and Technology 17:R175-R195.

Beeby, S. P., R. N. Torah, M. J. Tudor, P. Glynne-Jones, T. O’Donnell, C. R. Saha, and S. Roy. 2007. A micro electromagnetic generator for vibration energy harvesting. Journal of Micromechanics and Microengineering 17:1257-1265.

Benz, M., W. B. Euler, and O. J. Gregory. 2002. The role of solution phase water on the deposition of thin films of poly(vinylidene fluoride). Macromolecules 35:26822688.

Bodkhe, S., P. S. M. Rajesh, S. Kamle, and V. Verma. 2014. Beta-phase enhancement in polyvinylidene fluoride through filler addition: comparing cellulose with carbon nanotubes and clay. Journal of Polymer Research 21:434.

Chang, C., V. H. Tran, J. Wang, Y.-K. Fuh, and L. Lin. 2010. Direct-write piezoelectric polymeric nanogenerator with high energy conversion efficiency. Nano Letters 10:726-731.

Cheng, F., J. He, T. Yan, C. Liu, X. Wei, J. Li, and Y. Huang. 2016. Antibacterial and hemostatic composite gauze of N,O-carboxymethyl chitosan/oxidized regenerated cellulose. RSC Advances 6:94429-94436.

Costa, P., J. Silva, V. Sencadas, C. M. Costa, F. W. J. van Hattum, J. G. Rocha, and S. Lanceros-Mendez. 2009. The effect of fibre concentration on the $\alpha$ to $\beta$-phase transformation, degree of crystallinity and electrical properties of vapour grown carbon nanofibre/poly(vinylidene fluoride) composites. Carbon 47:2590-2599.

Doll, W. W., and J. B. Lando. 1970. Polymorphism of poly(vinylidene fluoride) IV. Structure of high-pressure-crystallized poly(vinylidene fluoride). Journal of Macromolecular Science Part B 4:889-896.

Doyle, J. J., and E. E. Dickson. 1987. Preservation of plant samples for DNA restriction endonuclease analysis. Taxon 36: 715-722.

El Achaby, M., F.-E. Arrakhiz, S. Vaudreuil, E.-M. Essassi, A. Qaiss, and M. Bousmina. 2013. Nanocomposite films of poly(vinylidene fluoride) filled with polyvinylpyrrolidone-coated multiwalled carbon nanotubes: Enhancement of $\beta$ polymorph formation and tensile properties. Polymer Engineering and Science 53:34-43.

Fu, R., S. Chen, Y. Lin, S. Zhang, J. Jiang, Q. Li, and Y. Gu. 2017. Improved piezoelectric properties of electrospun poly(vinylidene fluoride) fibers blended with cellulose nanocrystals. Materials Letters 187:86-88. 
Gradys, A., P. Sajkiewicz, S. Adamovsky, A. Minakov, and C. Schick. 2007. Crystallization of poly(vinylidene fluoride) during ultra-fast cooling. Thermochimica Acta 461:153-157.

Gregorio Jr., R., and R. C. Capitao. 2000. Morphology and phase transition of high melt temperature crystallized poly(vinylidene fluoride). Journal of Materials Science 35:299-306.

Han, J., C. Zhou, Y. Wu, F. Liu, and Q. Wu. 2013. Self-assembling behavior of cellulose nanoparticles during freeze-drying: Effect of suspension concentration, particle size, crystal structure, and surface charge. Biomacromolecules 14:1529-1540.

Hattori, T., M. Hikosaka, and H. Ohigashi. 1996. The crystallization behaviour and phase diagram of extended-chain crystals of poly(vinylidene fluoride) under high pressure. Polymer 37:85-91.

Imran-ul-haq, M., B. Tiersch, and S. Beuermann. 2008. Influence of polymer end groups on crystallization and morphology of poly(vinylidene fluoride) synthesized in homogeneous phase with supercritical carbon dioxide. Macromolecules 41:74537462.

Issa, A. A., M. Al-Maadeed, A. S. Luyt, M. Mrlik, and M. K. Hassan. 2016. Investigation of the physico-mechanical properties of electrospun PVDF/cellulose (nano)fibers. Journal of Applied Polymer Science 133(26):43594.

Lee, M., C.-Y. Chen, S. Wang, S. N. Cha, Y. J. Park, J. M. Kim, L.-J. Chou, and Z.-L. Wang. 2012. A hybrid piezoelectric structure for wearable nanogenerators. Advanced Materials 24:1759-1764.

Lopes, A. C., C. M. Costa, C. J. Tavares, I. C. Neves, and S. Lanceros-Mendez. 2011. Nucleation of the electroactive $\gamma$ phase and enhancement of the optical transparency in low filler content poly(vinylidene)/clay nanocomposites. Journal of Physical Chemistry C 115:18076-18082.

Lopes, A. C., C. Caparros, J. L. Gomez Ribelles, I. C. Neves, and S. Lanceros-Mendez. 2012. Electrical and thermal behavior of $\gamma$-phase poly(vinylidene fluoride)/ $\mathrm{NaY}$ zeolite composites. Microporous and Mesoporous Materials 161:98-105.

Lovinger, A. J. 1981. Unit Cell of the $\gamma$ Phase of Poly(vinylidene fluoride). Macromolecules 14:322-325.

Mandal, D., K. J. Kim, and J. S. Lee. 2012. Simple synthesis of palladium nanoparticles, $\beta$-Phase formation, and the control of chain and dipole orientations in palladiumdoped poly(vinylidene fluoride) thin films. Langmuir 28:10310-10317.

Martins, P., A. C. Lopes, and S. Lanceros-Mendez. 2014. Electroactive phases of poly(vinylidene fluoride): Determination, processing and applications. Progress in Polymer Science 39:683-706.

Milani, A., C. Castiglioni, and S. Radice. 2015. Joint experimental and computational investigation of the structural and spectroscopic properties of poly(vinylidene fluoride) polymorphs. Journal of Physical Chemistry B 119:4888-4897. 
Moon, R. J., A. Martini, J. Nairn, J. Simonsen, and J. Youngblood. 2011. Cellulose nanomaterials review: structure, properties and nanocomposites. Chemical Society Reviews 40:3941-3994.

Necas, D., and P. Klapetek. 2012. Gwyddion: an open-source software for SPM data analysis. Central European Journal of Physics 10:181-188.

Okita, Y., S. Fujisawa,T/ Saito, and A. Isogai. 2011. TEMPO-oxidized cellulose nanofibrils dispersed in organic solvents. Biomacromolecules 12:518-522.

Ouyang, J., C.-W. Chu, C. R. Szmanda, L. Ma, and Y. Yang. 2004. Programmable polymer thin film and non-volatile memory device. Nature Materials 3:918-922.

Pan, H., B. Na, R. Lv, C. Li, J. Zhu, and Z. Yu. 2012. Polar phase formation in poly(vinylidene fluoride) induced by melt annealing. Journal of Polymer Science Part B: Polymer Physics 50:1433-1437.

Patro, T. U., M. V. Mhalgi, D. V. Khakhar, and A. Misra. 2008. Studies on poly(vinylidene fluoride)-clay nanocomposites: Effect of different clay modifiers. Polymer 49:3486-3499.

Perez, R., M. Kral, and H. Bleuler. 2012. Study of polyvinylidene fluoride (PVDF) based bimorph actuators for laser scanning actuation at $\mathrm{kHz}$ frequency range. Sensors and Actuators A 183:84-94.

Rajesh, P. S. M., S. Bodkhe, S. Kamle, and V. Verma. 2014. Enhancing Beta-phase in PVDF through physicochemical modification of cellulose. Electronic Materials Letters 10:315-319.

Ramadan, K. S., D. Sameoto, and S. Evoy. 2014. A review of piezoelectric polymers as functional materials for electromechanical transducers. Smart Materials and Structures 23:033001.

Ribeiro, C., V. Sencadas, J. L. Gomez-Ribelles, and S. Lanceros-Mendez. 2010. Influence of processing conditions on polymorphism and nanofiber morphology of electroactive poly(vinylidene fluoride) electrospun membranes. Soft Matter 8:274-87.

Sacui, I. A., R. C. Nieuwendaal, D. J. Burnett, S. J. Stranick, M. Jorfi, C. Weder, E. J. Foster, R. T. Olsson, and J. W. Gilman. 2014. Comparison of the properties of cellulose nanocrystals and cellulose nanofibrils isolated from bacteria, tunicate, and wood processed using acid, enzymatic, mechanical, and oxidative methods. ACS Applied Materials and Interfaces 6:6127-6138.

Salimi A., and A. A. Yousefi. 2003. FTIR studies of beta-phase crystal formation in stretched PVDF films. Polymer Testing 22:699-704.

Scott, J. C., and L. D. Bozano. 2007. Nonvolatile memory elements based on organic materials. Advanced Materials 19:1452-1463.

Sencadas, V., R. Gregorio Jr., and S. Lanceros-Mendez. 2009. $\alpha$ to $\beta$ phase transformation and microestructural changes of PVDF films induced by uniaxial stretch. Journal of Macromolecular Science, Part B: Physics 48:514-525. 
Sencadas, V., P. Martins, A. Pitaes, M. Benelmekki, J. L. Gomez Ribelles, and S. LancerosMendez. 2011. Influence of ferrite nanoparticle type and content on the crystallization kinetics and electroactive phase nucleation of poly(vinylidene fluoride). Langmuir 27:7241-7249.

Sirohi, J., and I. Chopra. 2000. Fundamental understanding of piezoelectric strain sensors. Journal of Intelligent Material Systems and Structures 11:246-257.

Tamang, A., S. Kumar Ghosh, S. Garain, M. M. Alam, J. Haeberle, K. Henkel, D. Schmeisser, and D. Mandal. 2015. DNA-assisted $\beta$-phase nucleation and alignment of molecular dipoles in PVDF Film: A realization of self-poled bioinspired flexible polymer nanogenerator for portable electronic devices. ACS Applied Materials and Interfaces 7:16143-16147.

Ten, E., L. Jiang, and M. P. Wolcott. 2012. Crystallization kinetics of poly(3hydroxybutyrate-co-3-hydroxyvalerate)/cellulose nanowhiskers composites. Carbohydrate Polymers 90:541-550.

Wang, W., S. Zhang, L. O. Srisombat, T. R. Lee, and R. C. Advincula. 2011. Goldnanoparticle- and gold-nanoshell-induced polymorphism in poly(vinylidene fluoride). Macromolecular Materials and Engineering 296:178-184.

Wang, L., and S. Chen. 2016. Crystallization behaviors of poly(vinylidene fluoride) and poly(methyl methacrylate)-block-poly(2-vinyl pyridine) block copolymer blends Journal of Thermal Analysis and Calorimetry 125:215-230.

Weinhold, S., M. H. Litt, and J. B. Lando. 1980. The crystal structures of the $\gamma$ phase of Poly(vinylidene fluoride). Macromolecules 13:1178-1183.

Xu, X., F. Liu, L. Jiang, J. Y. Zhu, D. Haagenson, and D. P. Wiesenborn. 2013. Cellulose nanocrystals vs. cellulose nanofibrils: A Comparative study on their microstructures and effects as polymer reinforcing agents. ACS Applied Materials and Interfaces 5:2999-3009.

Yang, D., and E. L. Thomas. 1987. Effect of PMMA on the morphology and $\alpha \rightarrow \beta$ phase transition of oriented $\mathrm{PVF}_{2} / \mathrm{PMMA}$ blends. Journal of Materials Science Letters 6:593-598.

Yang, D. C., and Y. Chen. 1987. $\beta$-phase formation of poly(vinylidene fluoride) from the melt induced by quenching. Journal of Materials Science Letters 6:599-603.

Ye, H., W. Shao, and L. Zhen. 2013. Crystallization kinetics and phase transformation of poly(vinylidene fluoride) films incorporated with functionalized $\mathrm{BaTiO}_{3}$ nanoparticles. Journal of Applied Polymer Science 129:2940-2949.

Ying, L., E. T. Kang, and K. G. Neoh. 2002. Covalent immobilization of glucose oxidase on microporous membranes prepared from poly(vinylidene fluoride) with grafted poly(acrylic acid) side chains. Journal of Membrane Science 208:361-374.

Yoon, S., A. A. Prabu, K. J. Kim, and C. Park. 2008. Metal salt-induced ferroelectric crystalline phase in poly(vinylidene fluoride) films. Macromolecular Rapid Communications 29:1316-1321. 
Yu, S., W. Zheng, W. Yu, Y. Zhang, Q. Jiang, and Z. Zhao. 2009. Formation mechanism of $\beta$-phase in PVDF/CNT composite prepared by the sonication method.

Macromolecules 42:8870-8874.

Zheng, J., A. He, J. Li, and C. C. Han. 2007. Polymorphism control of poly(vinylidene fluoride) through electrospinning. Macromolecular Rapid Communications 28:2159-2162. 


\section{Appendix A: PVDF Films with Added Water}

In order to test the effect of water on the crystal structure of PVDF, a series of PVDF films were prepared by dissolving PVDF pellets (Sigma Aldrich 427152, MW 180,000) in dry N,N-dimethyl formamide (DMF) (Sigma Aldrich 1023752500 ) at $60^{\circ} \mathrm{C}$ to obtain a $10 \mathrm{wt} \%$ solution, and then adding a known amount of water (o to $5 \mathrm{wt} \%$ ). Solvent evaporation was carried out overnight at $100^{\circ} \mathrm{C}$. The films were characterized with FTIR spectroscopy. As shown in Figure A1[A], all films have a dominant $\gamma$ crystalline phase and the addition of water does not induce nucleation of $\beta$ phase. In a similar experiment, Benz et al. (2002) added water to the solvent prior to polymer dissolution and concluded that even though water affected the surface morphology, it did not induce $\beta$ phase nucleation due to rapid evaporation. Figure A1[B] shows that FTIR spectra from $3100-3600 \mathrm{~cm}^{-1}$, but none of the films, regardless of added water wt\%, show evidence of a hydroxyl-related band. The lack of the hydroxyl peak further supports the hypothesis that water rapidly evaporates from the polymer/solvent system and does not influence the film crystallization process. FTIR intensity ratios representative of PVDF crystalline phases, as function of water wt\%, are shown in Figure A1[C]. For this set of films, the $\mathrm{A}_{1275} / \mathrm{A}_{874}$ ratio was not plotted because no distinct peak was observed around $1275 \mathrm{~cm}^{-1}$. In agreement with other neat PVDF films obtained under similar processing conditions (primarily the solvent evaporation temperature), the films are predominantly $\gamma$ phase with some minority $\alpha$ phase. 
Figure A1. A. FTIR spectra of neat PVDF and PVDF films with water added to the solvent (series C). B. FTIR spectra in the $3100-3600 \mathrm{~cm}^{-1}$ range showing the lack of the hydroxyl band. C. Calculated intensity ratios for qualitative estimation of $\alpha, \beta$, and $\gamma$ phases in the composite films as function of added water wt\%.
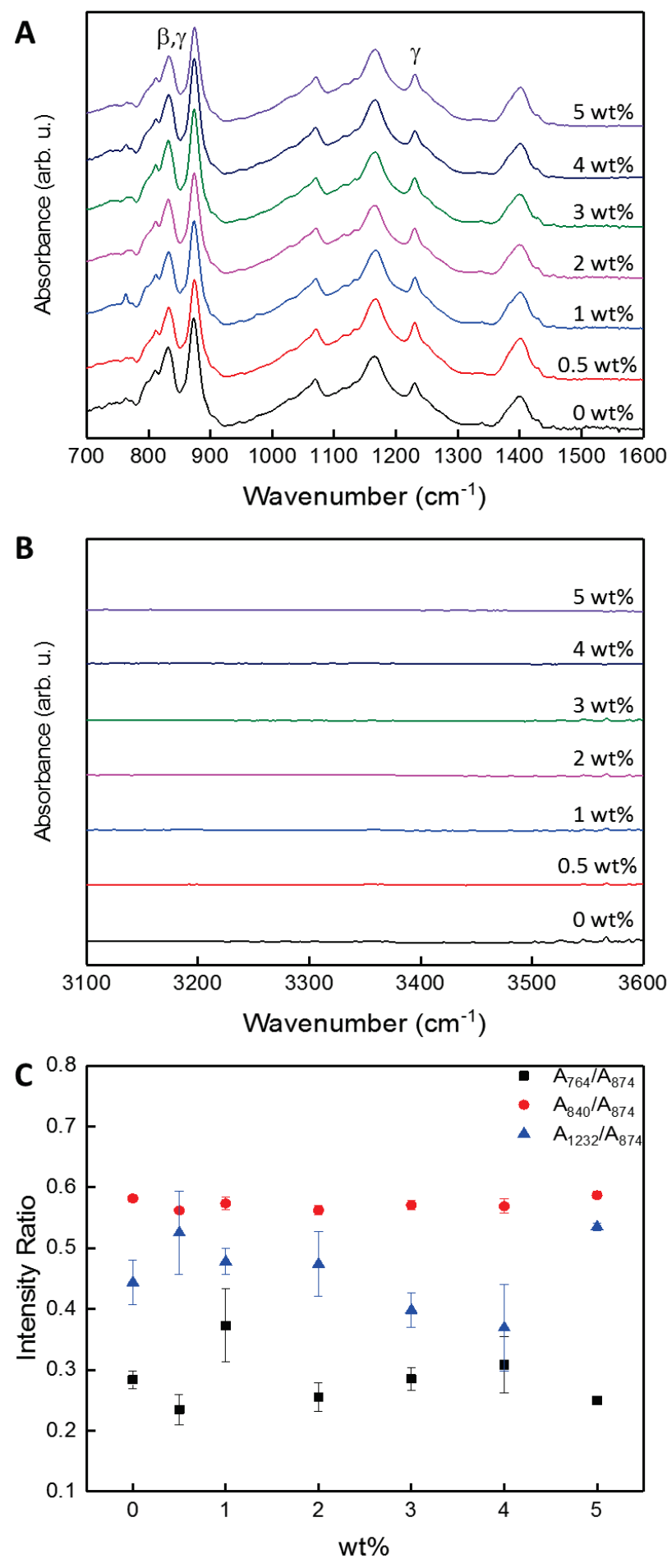


\section{Appendix B: Cellulose Nanofibril/PVDF Films Obtained from As-Received Cellulose Nanofibril Water Slurry}

A series of as-received cellulose nanofibril/PVDF composites films (series

D) were synthesized. When compared to series A, the as-received cellulose slurry was incorporated in the dissolved PVDF/DMF solution without solvent exchange. Solvent evaporation was carried out at $100^{\circ} \mathrm{C}$ overnight. The composite films were characterized with a variety of characterization techniques such as optical microscopy, SEM, XRD, FTIR, Raman, DSC, and their mechanical properties such as tensile strength and modulus were also measured with a DMA instrument. Details regarding these characterization techniques can be found in the main text of the report. Raman spectra were collected with a confocal Raman microscope (Horiba Xplora Plus) equipped with a $532 \mathrm{~nm}$ excitation laser. Incident light ( 0.4 $\mathrm{mW}$ ) was focused onto the surface of the samples with a $100 \times$ objective. The acquisition time for each point spectrum was $120 \mathrm{sec}$, and multiple spectra were collected for each sample. The instrument was calibrated with respect to the first order silicon peak at $520.6 \mathrm{~cm}^{-1}$.

Optical images of the films with o, 1, 2, 4 and 8 wt\% cellulose nanofibril loading (from left to right) are shown in Figure B1[A]. Lowmagnification cross-polarized images of the films, under transmission mode, are shown in Figures B1[B]-[F]. For the neat and $1 \mathrm{wt} \%$ films, the surface morphology looks similar and consists of voids and interconnected spherulites, whereas for the higher wt\% doped films, the surfaces become smoother but with visible cellulose fibrils on the surface. Higher magnification SEM images of the surfaces are shown in Figure B2. All SEM images were collected on the free (rough) facet of the PVDF films. In agreement with the optical images, the neat PVDF film consists of interconnected spherulites with diameters in the order of $\sim 10 \mu \mathrm{m}$ with noticeable voids between some of them. As the amount of cellulose nanofibrils increases, both the average spherulite size and the void space decrease, up to at least $4 \mathrm{wt} \%$. The $8 \mathrm{wt} \%$ film has small, well-connected spherulites, but small voids are present, likely due to the inhomogeneous mixing of the cellulose and the relatively large amount of water present in PVDF film during the evaporation process. 
Figure B1. A. Picture of 0, 1, 2, 4 and 8 wt\% cellulose nanofibril/PVDF films (series D). Increasing cellulose nanofibril wt\% from left to right. $20 \times$ magnification optical crossed-polarized images of B. 0 wt\%, C. 1 wt\%, D. 2 wt\%, E. 4 wt\%, and F. 8 wt\% cellulose nanofibril/PVDF composites (series D).
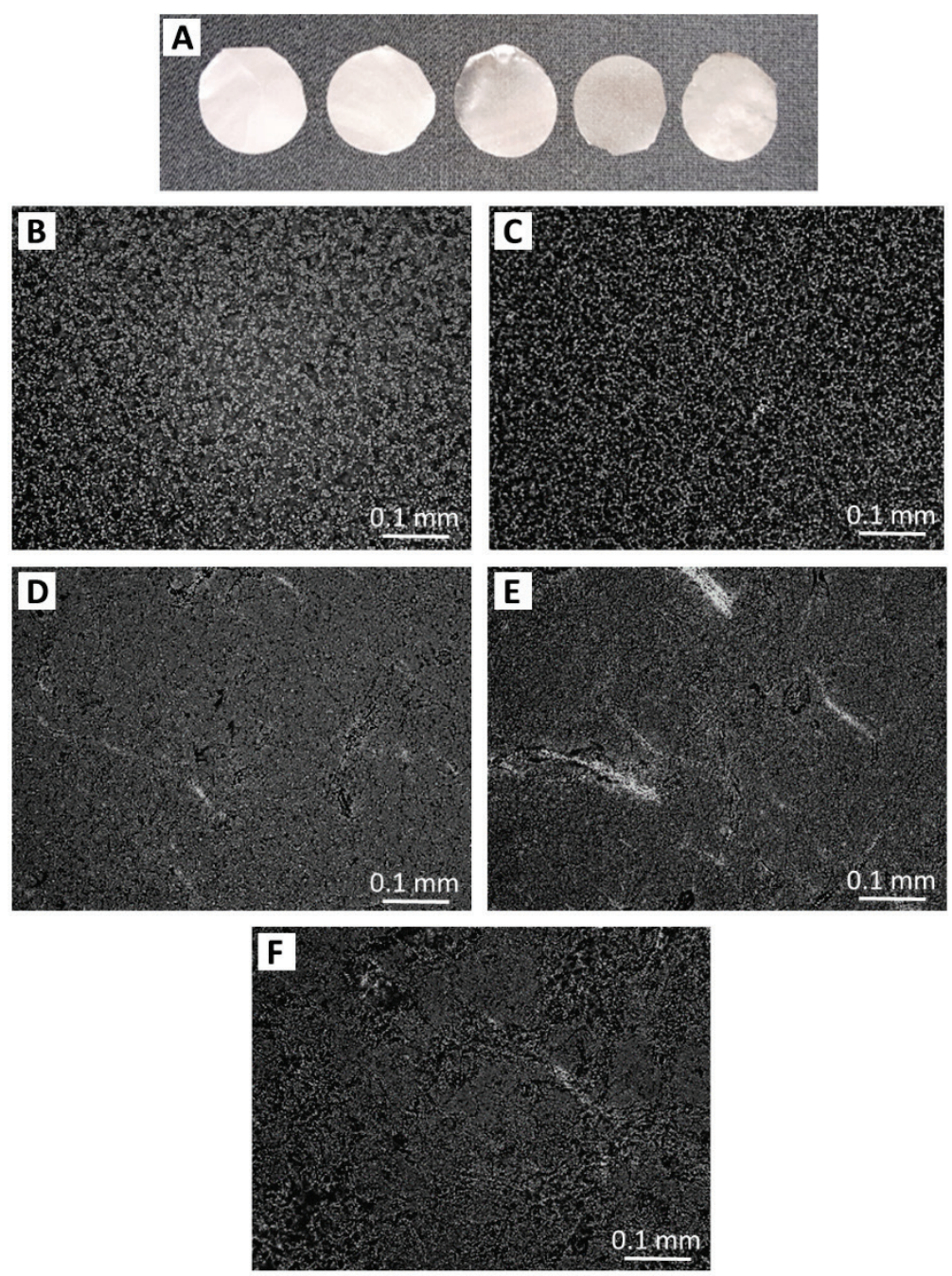

XRD was carried out to determine the crystalline phase of the neat and composites films. As shown in Figure B3, all the diffraction patterns show two major peaks at approximately $21.6^{\circ}$ and $23.6^{\circ}$, which correspond to the (020) and (110) reflections of $\gamma$ PVDF, and a smaller peak at $\sim 46^{\circ}$, which is also assigned to the (210) $\gamma$ reflection (Martins et al. 2014). For the $4 \mathrm{wt} \%$ film, a small peak at approximately $31^{\circ}$ can be assigned to a weak $\alpha$ (100) reflection, indicating the presence of at least two crystalline phases in the particular film. For the 4 and 8 wt\% films, the shoulder emerging on the higher side of the $23.6^{\circ}$ peak is likely related to the increasing amount of cellulose on the film surface. Further, the broadening and loss of contrast of the XRD peaks for the highest 
loaded sample could be related to increased disorder and loss of crystallinity due to inhomogeneous incorporation of cellulose.

Figure B2. SEM images of film surface for A. Neat PVDF, B. 1 wt\%, C. 2 wt\%, D. 4 wt\%, and E. 8 wt\% cellulose nanofibril/PVDF composites (series D).
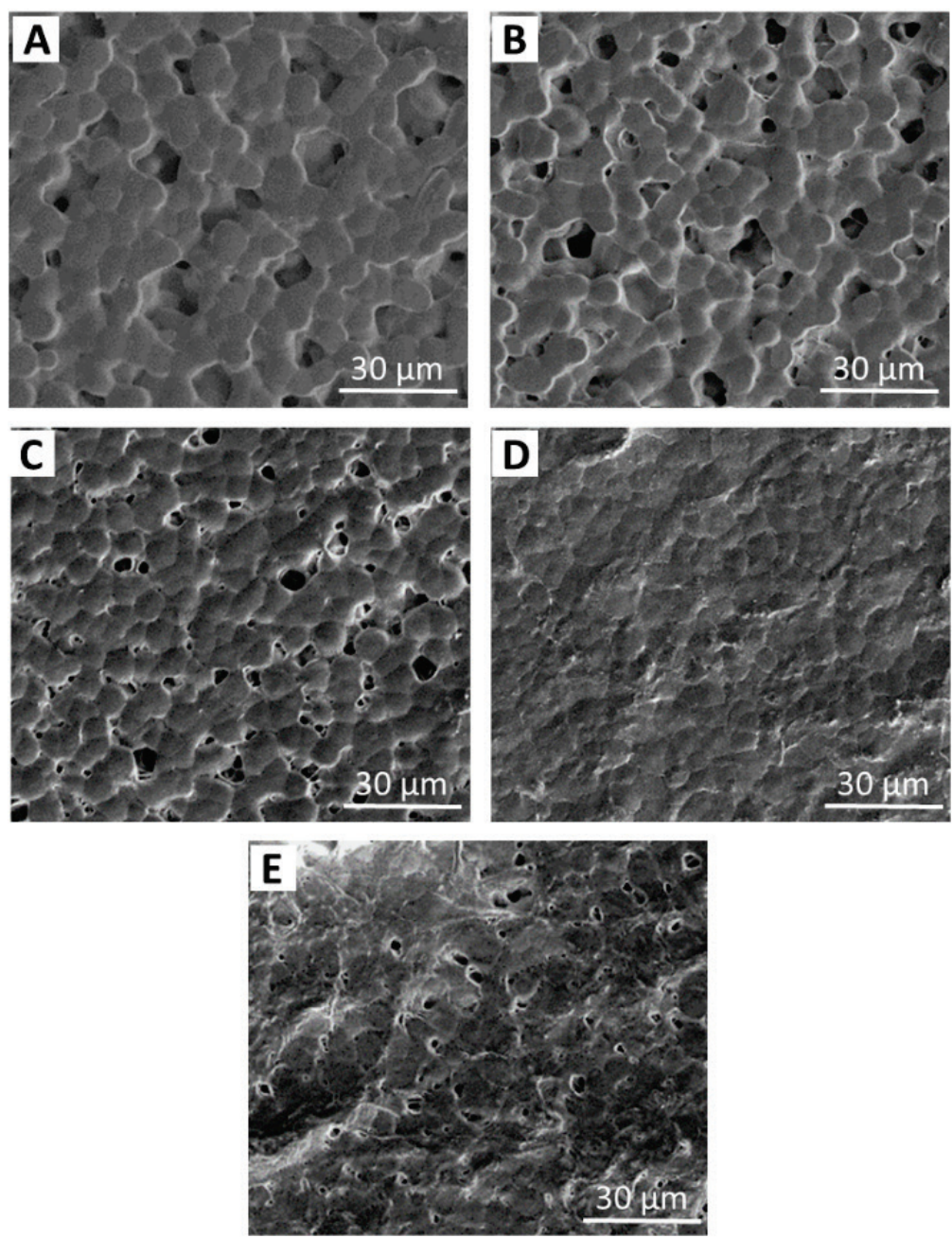

The FTIR spectra of the neat PVDF and composite films are shown in Figure B4[A]. For the neat film, the peaks at $811 \mathrm{~cm}^{-1}$ and $1232 \mathrm{~cm}^{-1}$ indicate that the dominant crystalline phase is the $\gamma$ phase. Using equation (1), the $\gamma$ phase crystalline fraction of the neat film was estimated to be $F(\gamma) \sim 92 \%$. The addition of cellulose nanofibrils does not dramatically alter the FTIR spectra of the composites, but slightly reduces $F(\gamma)$ (see Table B1). A closer look at the FTIR spectra shows that for approximately 2 wt\% loading, a shoulder at $\sim 1275 \mathrm{~cm}^{-1}$ becomes more prominent, corresponding to the presence of $\beta$ phase. Similarly, a peak at $764 \mathrm{~cm}^{-1}$ indicates the emergence of $\alpha$ phase. Therefore, at higher loadings, the as-received nanocellulose/PVDF 
composites are mainly $\gamma$ PVDF with a small $\alpha$ and $\beta$ phase content. Qualitative information regarding the $\alpha, \beta$, and $\gamma$ phases are obtained from the FTIR absorbance intensity ratios $\mathrm{A}_{764} / \mathrm{A}_{874}, \mathrm{~A}_{1275} / \mathrm{A}_{874}, \mathrm{~A}_{840} / \mathrm{A}_{874}$, and $\mathrm{A}_{1232} / \mathrm{A}_{874}$ where $\mathrm{A}_{764}, \mathrm{~A}_{1275}, \mathrm{~A}_{840}, \mathrm{~A}_{874}$, and $\mathrm{A}_{1232}$ are the measured absorbances at $764 \mathrm{~cm}^{-1}, 1275 \mathrm{~cm}^{-1}, 840 \mathrm{~cm}^{-1}, 874 \mathrm{~cm}^{-1}$, and $1232 \mathrm{~cm}^{-1}$, respectively (Imran-ul-haq et al. 2008). As shown in Figure B4[B], $\mathrm{A}_{764} / \mathrm{A}_{874}$ reaches a maximum at $4 \mathrm{wt} \%$, whereas $\mathrm{A}_{840} / \mathrm{A}_{874}$, and $\mathrm{A}_{1275} / \mathrm{A}_{874}$ increase up to $2 \mathrm{wt} \%$ and either plateau or slightly decrease beyond that. On the other hand, $\mathrm{A}_{1232} / \mathrm{A}_{874}$ decreases for loadings higher than $2 \mathrm{wt} \%$. Overall, addition of the as-received cellulose water-based slurry in PVDF has a modest effect in nucleating both polar and non-polar phases in $\gamma$ PVDF.

Figure B3. XRD patterns for cellulose nanofibril/PVDF composites (series D).

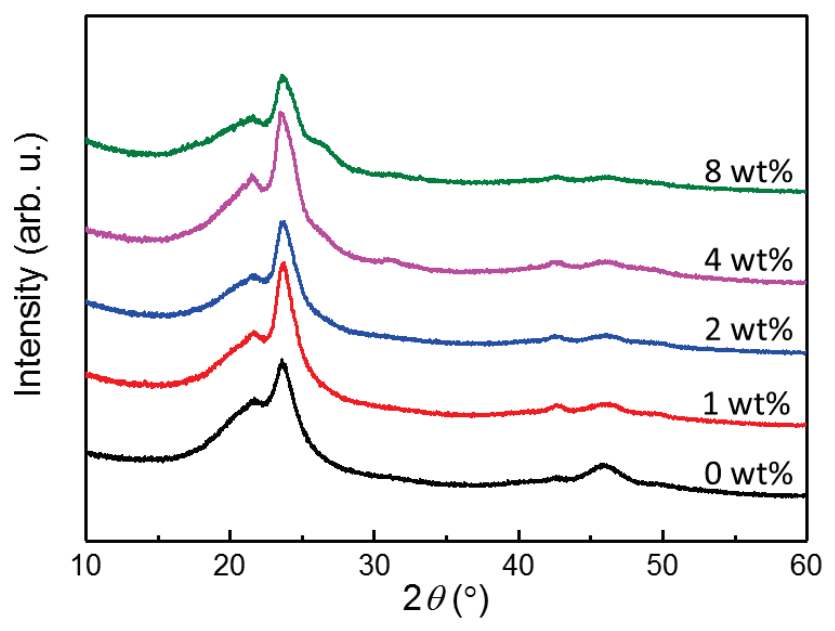

The FTIR spectra in the $3100-3600 \mathrm{~cm}^{-1}$ range of Figure $\mathrm{B} 5[\mathrm{~A}]$ show the gradual emergence of a broad absorption peak associated with hydroxyl groups as the amount of cellulose incorporated in the films increases. Cellulose is both hydrophyllic and hygroscopic and likely facilitates retention of water during the solvent evaporation process. It is known that hydrated or hygroscopic salts induce phase transformations in PVDF. For example, in Benz et al. (2002), hygroscopic salts added to PVDF dissolved in an acetone/DMF solvent mixture induced some transformation from $\gamma$ to $\beta$ PVDF. It is hypothesized that strong hydrogen-bonding interaction of water molecules towards the fluorine cations of PVDF aligns the $-\mathrm{CH}_{2} /-\mathrm{CF}_{2}$ dipoles in the all trans (TTTT) $\beta$ configuration. Figure $\mathrm{B}_{5}[\mathrm{~B}]$ shows the absorption peaks related to the symmetric and asymmetric stretching vibration $\mathrm{CH}_{2}$ bands of PVDF. For the neat film, the bands are located at $\sim 2981$ and 3023 $\mathrm{cm}^{-1}$, respectively. For the 2 and $4 \mathrm{wt} \%$ films, both of the peaks shift to slightly 
higher wavenumbers (at 2983 and $3025 \mathrm{~cm}^{-1}$, respectively) indicating strong interfacial interactions between the dopants and the PVDF matrix.

Figure B4. A. FTIR spectra of neat PVDF and cellulose nanofibril/PVDF composites (series D). B. Calculated intensity ratios for qualitative estimation of $\alpha, \beta$, and $\gamma$ phases in the composite films as function of oxidized cellulose wt\%.
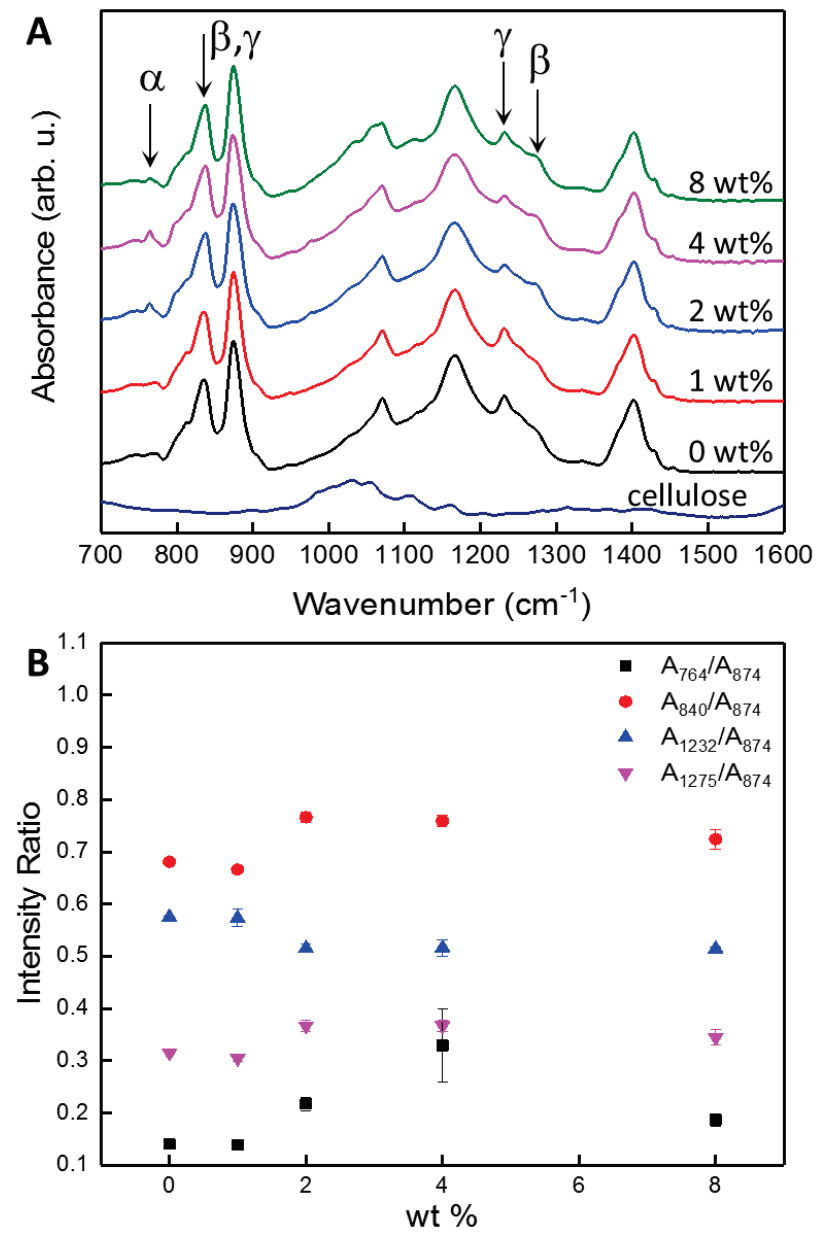

The coexistence of at least two crystalline phases in the composites films was also verified with high-magnification SEM imaging and micro-Raman spectroscopy. Figure B6[A] shows the typical morphology of the $4 \mathrm{wt} \%$ film revealing the presence of two morphologically distinct phases, i.e., smooth, oval-shaped spherulites corresponding to $\gamma$ phase surrounded by areas with radial (dendritic) topography corresponding to $\alpha$ phase PVDF.

Micro Raman spectra collected from film locations similar to those of Figure B6[A] are shown in Figure B6[B] and are labeled as "mainly $\alpha$ and $\gamma$ " and "mainly $\gamma$ and $\alpha$." For comparison, the micro Raman spectra from a commercial, optimized piezoelectric PVDF film are also included and 
labeled as "mainly $\beta$ and $\alpha$. ." As shown from the cluster of Raman peaks centered around $850 \mathrm{~cm}^{-1}$, the $\beta$ peak located at $\sim 840 \mathrm{~cm}^{-1}$ is slightly higher than the $\gamma$ peak at $\sim 835 \mathrm{~cm}^{-1}$, whereas the characteristic $\alpha$ peak is at a lower wavenumber $\left(\sim 795 \mathrm{~cm}^{-1}\right)$. All three spectra reveal that the films have both a majority and minority crystalline phase and are not purely one crystalline phase. However, quantification of the crystalline phases is not possible with Raman spectroscopy.

Figure B5. FTIR spectra of neat PVDF and cellulose nanofibril/PVDF composites (series D) A. Spectra in the $3100-3600 \mathrm{~cm}^{-1}$ range showing the emergence of the hydroxyl band with increasing cellulose wt\%. B. Spectra in the $2940-3060 \mathrm{~cm}^{-1}$ range showing $\mathrm{CH}_{2}$ vibrational peaks as function of cellulose wt\%.
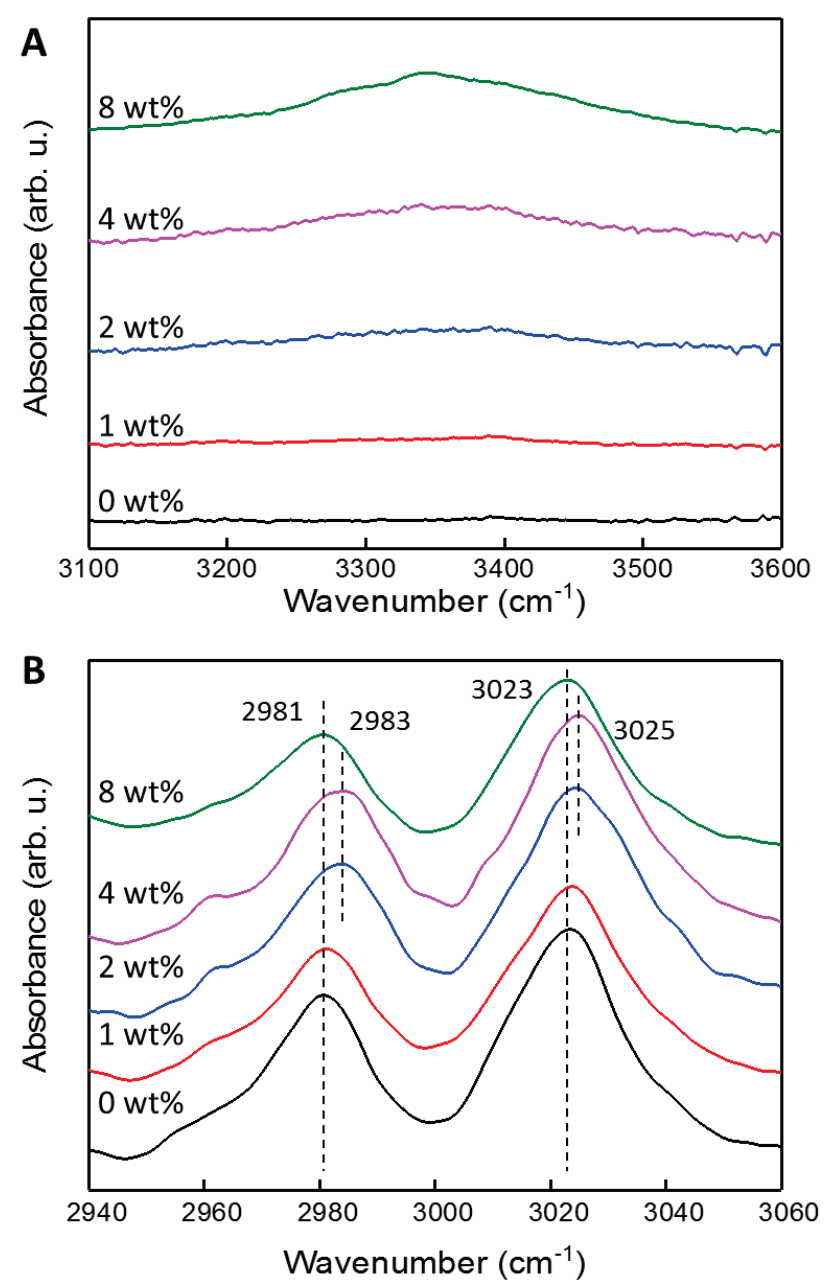
Figure B6. A. High-resolution SEM image showing the surface morphology of the 4 wt\% composite film, which consists of at least two morphologically distinct phases. B. Micro Raman spectra collected at locations similar to the ones shown in A ("mainly $\alpha$ and $\gamma$ " and "mainly $\gamma$ and $\alpha$ " spectra). The spectrum labeled "mainly $\beta$ and $\alpha$ " was obtained from a commercial piezoelectric PVDF film.
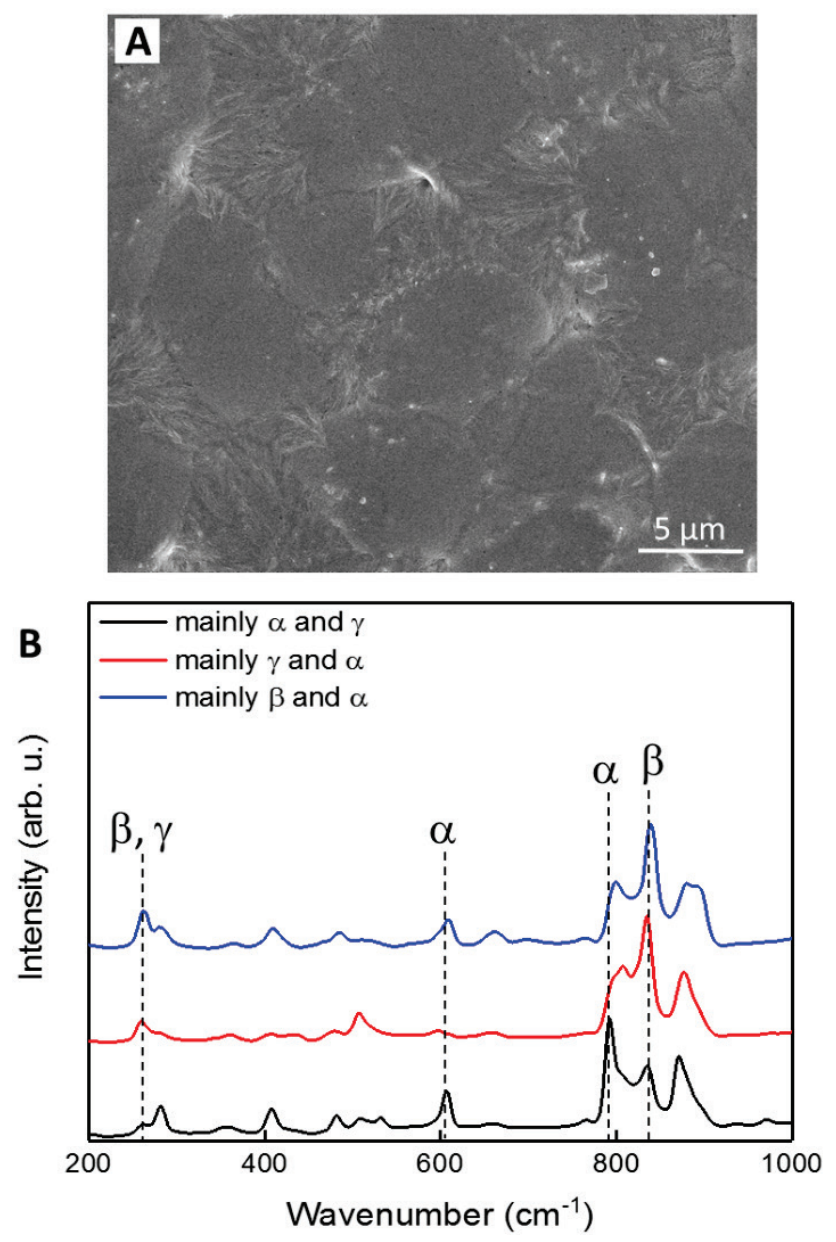

The melting behavior of the composites was evaluated with DSC and is shown in Figure B7. Relevant parameters such as the heat of fusion $\left(\Delta H_{f}\right)$, peak melting temperature $\left(T_{m}\right)$, and percent crystallinity $\left(X_{c}\right)$ are listed in Table B1. The addition of cellulose nanofibrils leads to reduction in the crystallinity of the composites with respect to the neat film, which also shifts $T_{m}$ to a lower temperature. In semicrystalline polymers, the melting temperature depends not only on crystallinity but also on the phases present in the film. For PVDF, it has been reported that the melting temperatures for the $\alpha$ and $\beta$ phases are in the $167-172^{\circ} \mathrm{C}$ range, and the $\gamma$ phase in the $179-180^{\circ} \mathrm{C}$ when obtained by crystallization from the melt, and even higher from $\alpha$ to $\gamma$ transformation (Martins et al. 2014). Similarly, the molecular weight of PVDF also affects its melting temperature as do defects 
due to processing conditions or type of additive. Decrease in crystallinity and melting temperature has been observed in other semicrystalline polymers doped with nanocellulose; for example, polyethylene oxide (PEO) has been attributed to confinement effect of the nano dopants that obstructs chain diffusion along the crystal growth front, resulting in thin spherulite lamellar thickness (Xu et al. 2013; Ten et al. 2012).

Figure B7. DSC showing the melting behavior of the neat PVDF, and the as-received cellulose nanofibril/PVDF composites (series D).

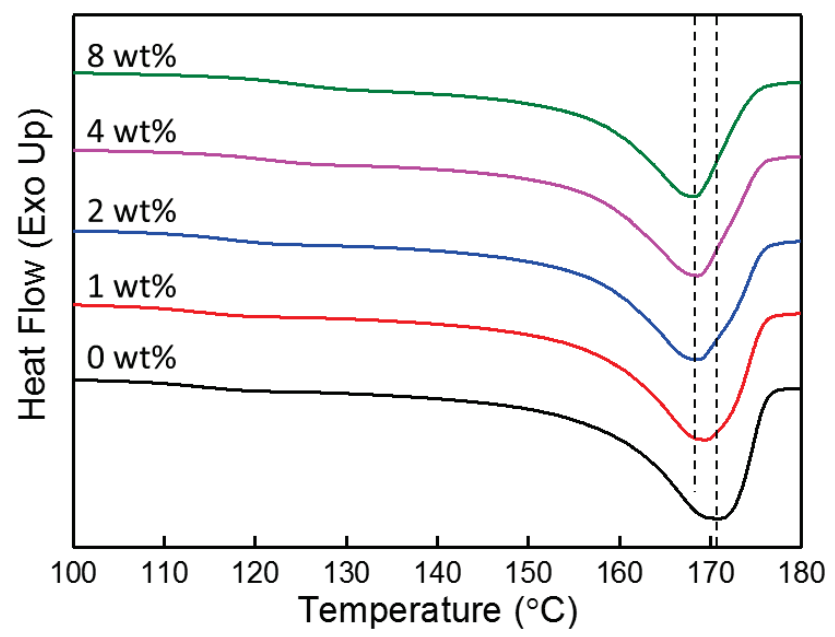

Table B1. FTIR crystalline phase fraction and DSC results for as-received cellulose nanofibril/PVDF films (Series D).

\begin{tabular}{|l|l|l|l|l|l|}
\hline & wt\% & \multicolumn{1}{|c|}{$\mathrm{F}(\gamma)$} & \multicolumn{1}{|c|}{$\Delta H_{f}(\mathrm{~J} / \mathrm{g})$} & \multicolumn{1}{|c|}{$X_{c}(\%) *$} & $T_{m}$ \\
\hline \multirow{4}{*}{ Series D } & 0 & 0.92 & 53.6 & 51.2 & 170.9 \\
\cline { 2 - 6 } & 1 & 0.92 & 52.2 & 50.4 & 169.4 \\
\cline { 2 - 6 } & 2 & 0.89 & 49.3 & 48.1 & 168.6 \\
\cline { 2 - 6 } & 4 & 0.84 & 47.2 & 47.0 & 168.6 \\
\cline { 2 - 6 } & 8 & 0.90 & 46.0 & 47.8 & 168.2 \\
\hline
\end{tabular}

The tensile properties (tensile strength, modulus, and \% elongation at break) of the composites are listed in Table B2. The tensile strength of the $1 \mathrm{wt} \%$ film shows a significant increase over that of the neat film but, with increasing loading, the tensile strength is reduced and reaches a minimum value for the 8 wt\% film. On the other hand, the tensile modulus of the composites shows large variations, likely due to film inhomogeneity, and reaches a minimum value for the $8 \mathrm{wt} \%$ film. Finally, the elongation at break measurements reveals loss of ductility in the composite films over that of the neat film. 
Table B2. Tensile properties of as-received cellulose nanofibril/PVDF films (Series D).

\begin{tabular}{|l|l|l|l|l|}
\hline & \multicolumn{1}{|c|}{$\begin{array}{c}\text { Tensile } \\
\text { strength } \\
\text { wt\% }\end{array}$} & $\begin{array}{c}\text { Tensile } \\
\text { Modulus } \\
(\mathrm{MPa})\end{array}$ & $\begin{array}{c}\text { Elongation } \\
\text { at break } \\
(\%)\end{array}$ \\
\hline \multirow{4}{*}{ Series D } & 0 & $35.7 \pm 1.7$ & $1083 \pm 71$ & $16.6 \pm 2.9$ \\
\cline { 2 - 5 } & 1 & $68.8 \pm 3.4$ & $824 \pm 320$ & $12.2 \pm 2.4$ \\
\cline { 2 - 5 } & 2 & $36.2 \pm 2.3$ & $1016 \pm 568$ & $5.4 \pm 0.7$ \\
\cline { 2 - 5 } & 4 & $41.3 \pm 10.6$ & $704 \pm 297$ & $6.8 \pm 0.7$ \\
\cline { 2 - 6 } & 8 & $20.8 \pm 2.9$ & $339 \pm 133$ & $5.4 \pm 1.1$ \\
\hline
\end{tabular}




\section{Appendix C: Melting and Recrystallization of Cellulose Nanofibril/PVDF Composites - Study 1}

It has been reported that melting and recrystallization of PVDF nanocomposites can yield $\alpha$ to $\gamma$ phase transformation (Lopes et al. 2012). Hence, some of the as-received cellulose nanofibril/PVDF films from series D were melted at $200^{\circ} \mathrm{C}$ for $1 \mathrm{hr}$ and then cooled down at room temperature. Figure $\mathrm{C} 1$ shows polarized optical microscopy images from the thermally treated films. As shown in Figure $\mathrm{C}_{1}[\mathrm{~A}]$, the neat PVDF film has highly birefringence spherulites that are indicative of $\alpha$ phase PVDF. As the amount of cellulose in the composites increases, the size of the spherulites decreases. In accordance with the optical images, higher resolution SEM images (see Figure C2) verify the presence of $\alpha$ spherulites that become smaller and more difficult to distinguish for the 4 and $8 \mathrm{wt} \%$ samples. The FTIR spectra from the thermally treated samples are shown in Figure $\mathrm{C}_{3}$ and unambiguously verify that all the films, regardless of cellulose wt\%, have transformed into the $\alpha$ phase. 
Figure C1. 20x magnification optical crossed-polarized images of A. 0 wt\%, B. 1 wt\%, C. 2 wt\%, D. 4 wt\%, and E. 8 wt\% cellulose nanofibril/PVDF composites (series D) after thermal treatment at $200^{\circ} \mathrm{C}$.
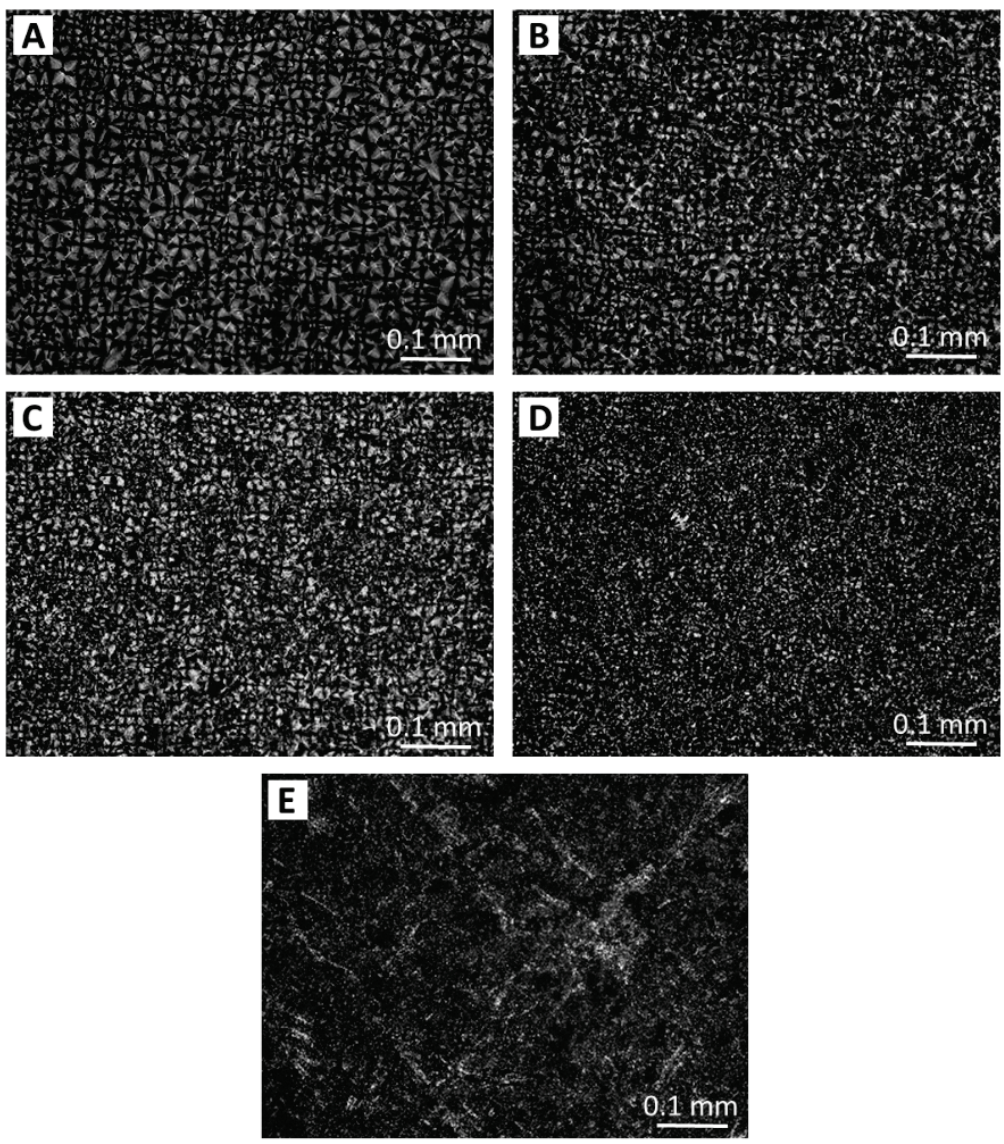
Figure C2. SEM images of the film surface for A. Neat PVDF, B. 1 wt\%, C. 2 wt\%, D. 4 wt\%, and E. 8 wt\% cellulose nanofibril/PVDF composites (series D) after thermal treatment at $200^{\circ} \mathrm{C}$.
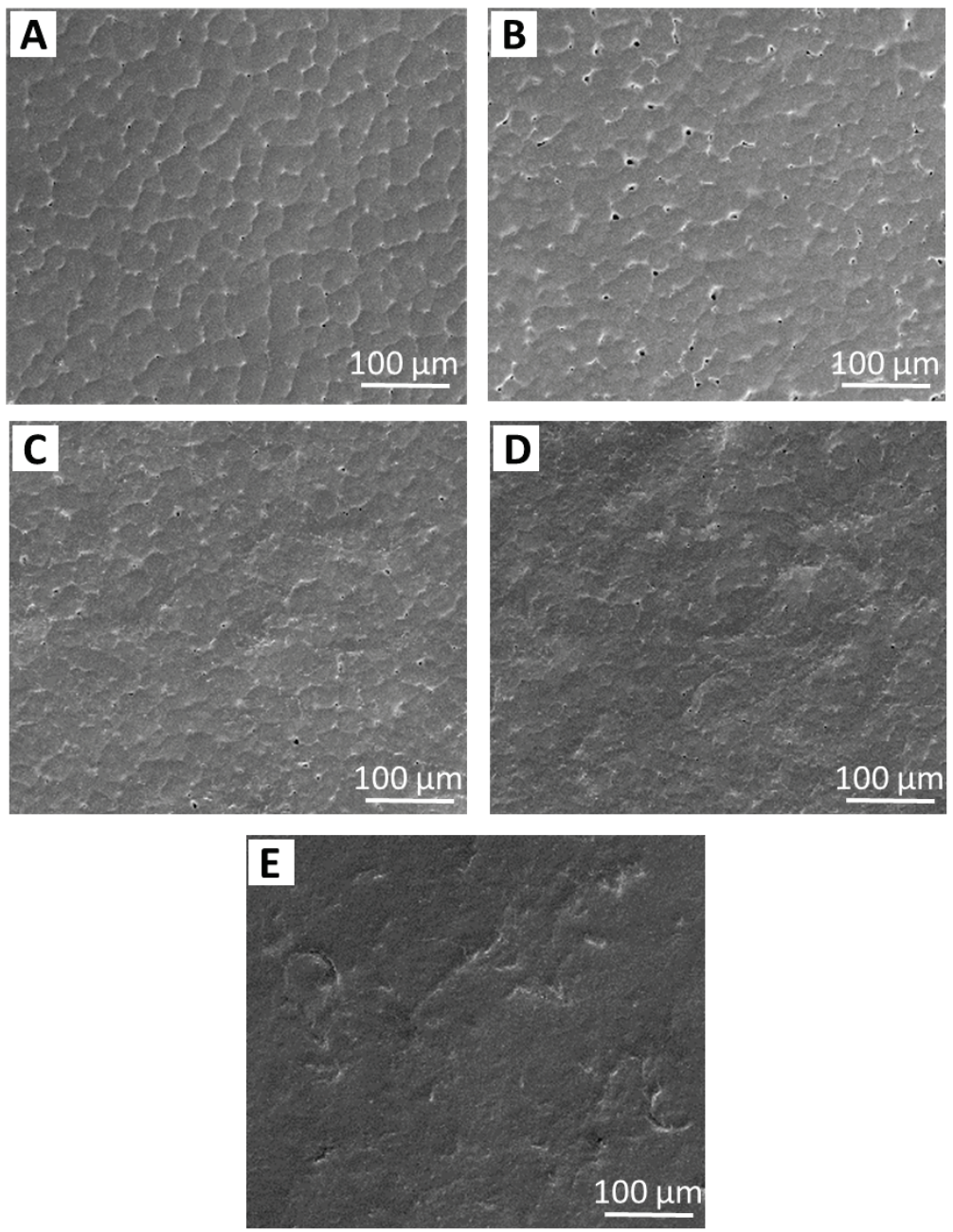

Figure C3. FTIR spectra of neat PVDF and cellulose nanofibril/PVDF composites (series D) after thermal treatment at $200^{\circ} \mathrm{C}$.

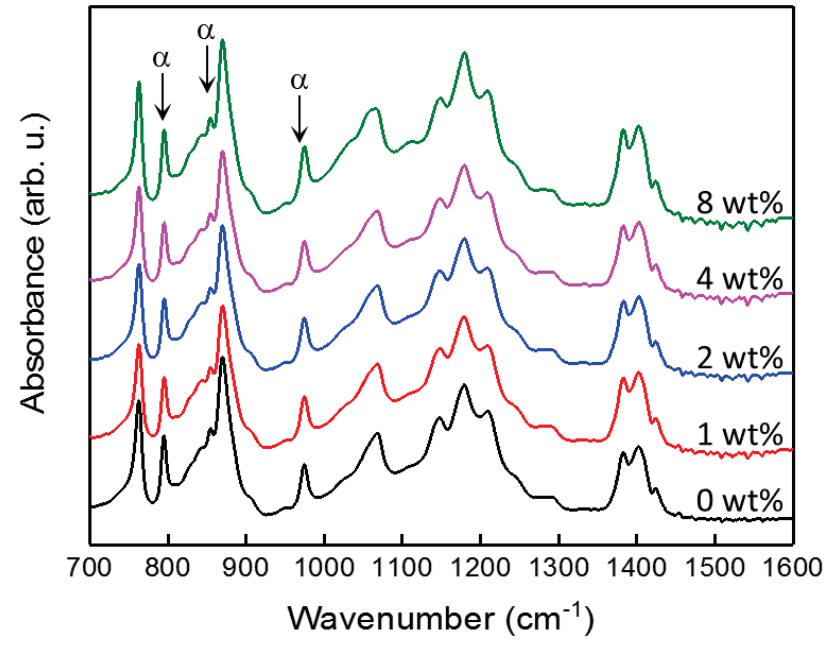




\section{Appendix D: Melting and Recrystallization of Cellulose Nanofibril/PVDF composites - Study \\ 2}

In this study, cellulose nanofibril/PVDF films were obtained by dissolving PVDF in dry DMF and dispersing solvent-exchanged nanocellulose. The solutions were cast onto clean glass plates and placed in a preheated oven $\left(17 \mathrm{O}, 180,190,200,210\right.$, and $\left.220^{\circ} \mathrm{C}\right)$ for $20 \mathrm{~min}$ to ensure solvent evaporation and melting. Next, the films were removed from the oven and allowed to cool down to room temperature. The FTIR spectra of the neat and $5 \mathrm{wt} \%$ cellulose nanofibril doped PVDF films are shown in Figure D1[A] and [B] respectively. In general, the neat films crystalize in a predominantly $\alpha$ phase, whereas the doped films crystalize in the $\gamma$ phase with the exception of the film treated at $220^{\circ} \mathrm{C}$.

Figure D1. FTIR spectra of A. neat PVDF films, B. 5 wt\% cellulose nanofibril/PVDF composites as function of melting temperature.
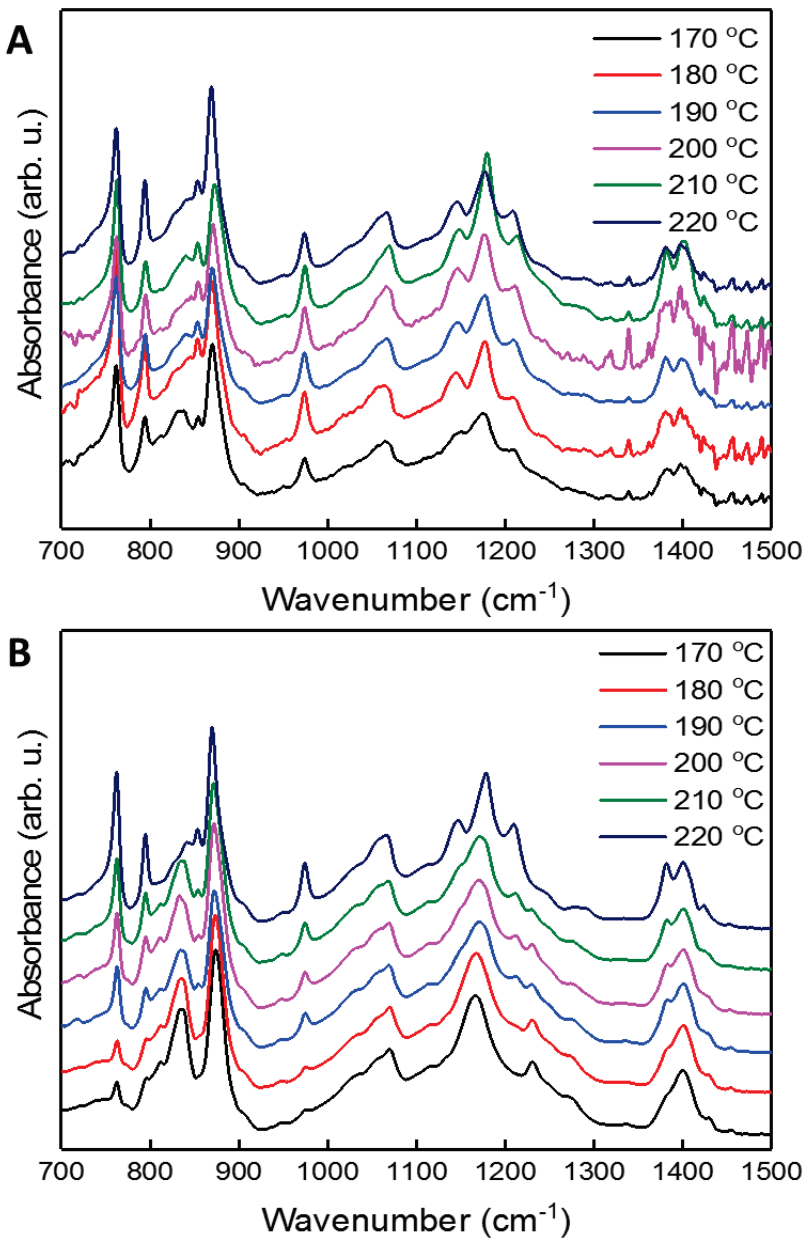


\section{Appendix E: DNA/PVDF Composites}

In an attempt to replicate the work of Tamang et al. (2015), a $1.5 \mathrm{wt} \%$ and a $0.25 \mathrm{wt} \%$ E.coli extracted DNA/PVDF composite film and a $2 \mathrm{wt} \%$ commercial DNA/PVDF film were synthesized. The lyophilized commercial DNA was genomic type XV from calf thymus and was obtained from Sigma Aldrich (D4522). Briefly, PVDF pellets (Sigma Aldrich 427152) were dissolved in distilled reagent grade DMF (Sigma Aldrich 319937). E. coli extracted DNA dissolved in water was then added to the PVDF/DMF solution, and solvent evaporation was carried out at $60^{\circ} \mathrm{C}$ overnight. The commercial DNA was suspended first in DMF and then added to the dissolved PVDF.

A detailed description of the $E$. coli DNA extraction protocol is given below (Doyle and Dickson 1987):

1. Transfer $50 \mathrm{~mL}$ of the overnight E. coli culture (grown in LB medium) to a $50 \mathrm{~mL}$ tube and centrifuge at a maximum speed for $5 \mathrm{~min}$ to pellet the cells.

2. Discard the supernatant.

3. Re-suspend the cell pellet in $4 \mathrm{~mL}$ of CTAB lysis buffer and vortex to completely re-suspend cell pellet. CTAB buffer composition: $2 \%$ cetyl trimethylammonium bromide, $1 \%$ polyvinyl pyrrolidone, $100 \mathrm{mM}$ Tris$\mathrm{HCl}, 1.4 \mathrm{M} \mathrm{NaCl}, 20 \mathrm{mM}$ EDTA.

4. Incubate $3 \mathrm{hr}$ at $55^{\circ} \mathrm{C}$.

5. Add an equal volume of chloroform/isoamyl alcohol (24:1) and mix well by inverting the tube until the phases are completely mixed.

6. Centrifuge for $5 \mathrm{~min}$ at room temperature (all spins are performed at room temperature unless indicated otherwise).

7. Carefully transfer the upper aqueous phase to a new tube.

8. To precipitate DNA, add 2.5 or 3 volume of cold isopropyl alcohol and ammonium acetate (7.5 M stock, $\mathrm{pH} 7.7$ ) to a final concentration of 2.5 $\mathrm{M}$ (store ethanol at $-20^{\circ} \mathrm{C}$ freezer) and mix gently (DNA precipitation can be visible).

9. Incubate the tube at $-20^{\circ} \mathrm{C}$ for 30 min or more.

10. Spin at max speed for $10 \mathrm{~min}$ at $4^{\circ} \mathrm{C}$.

11. Discard the supernatant and rinse the DNA pellet with $2 \mathrm{~mL} 70 \%$ ethanol (stored at $-20^{\circ} \mathrm{C}$ ).

12. Spin at max speed for 2 min. Carefully discard the supernatant.

13. Discard the supernatant and rinse the DNA pellet with $2 \mathrm{~mL} \mathrm{100 \%}$ ethanol (stored at $-20^{\circ} \mathrm{C}$ ). 
14. Spin at max speed for 2 min. Carefully discard the supernatant and airdry the DNA pellet (tilt the tube a little bit on paper towel). For faster results, dry the tube at $37^{\circ} \mathrm{C}$ incubator.

The films were examined with FTIR spectroscopy with the obtained spectra shown in Figure D1. Similar to other PVDF nanocomposites, the films exhibit a majority $\gamma$ phase. For the 2 wt\% commercial DNA, researchers observe a shoulder at $\sim 1275 \mathrm{~cm}^{-1}$ as well as a small shift of the peak at $838 \mathrm{~cm}^{-1}$ to higher wavenumbers, indicative of $\beta$ phase formation. It is therefore likely that adding more commercial DNA to PVDF will induce a change in its crystalline phase. It is worth noting that the amount of DNA added to the PVDF films was not reported in Tamang et al. (2015).

Figure E1. FTIR spectra of neat PVDF, 0.25 wt\% and 1.5 wt\% DNA/PVDF, and 2 wt\% commercial DNA/PVDF composites.

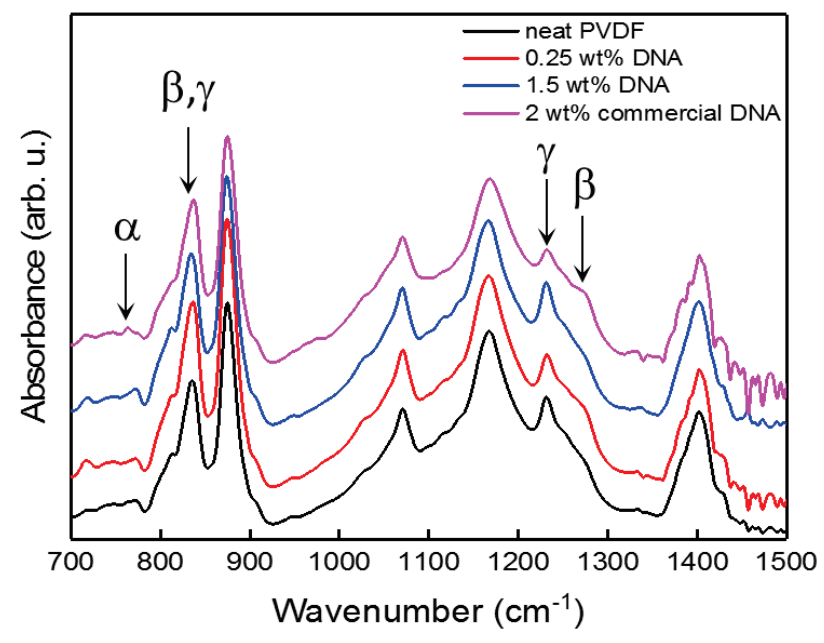




\section{Symbols and Acronyms}

The following symbols and acronyms are used throughout the report.

$\alpha$ - alpha, PVDF crystalline phase

$\beta$ - beta, PVDF crystalline phase

$\gamma$-gamma, PVDF crystalline phase

$\delta$ - delta, PVDF crystalline phase

$\varepsilon-$ epsilon, PVDF crystalline phase

$\theta$ - theta, used to describe diffraction angle in X-ray diffraction

AFM - Atomic Force Microscopy

$\mathrm{CH}$ - Methine

$\mathrm{CH}_{2}$ - Methylene

$\mathrm{COO}^{-}$- Deprotonated carboxyl

$\mathrm{COOH}$ - Carboxyl

$\mathrm{COO}^{-} \mathrm{Na}^{+}$or $\mathrm{COONa}$ - Sodium Carboxylate

COS - Carbonyl sulfide

$\mathrm{COSO}_{3}$ - Organosulfate

CTAB - Cetyl Trimethylammonium Bromide

DI - Deionized

DMA - Dynamic Mechanical Analysis

DMF - N, N-Dimethylformamide, an organic solvent

DMSO - Dimethyl sulfoxide, an organic solvent

DNA - Deoxyribonucleic Acid

DSC - Differential Scanning Calorimetry 
FTIR - Fourier Transform Infrared

g-grams

$\mathrm{hr}-$ hour

LB - Lysogeny broth, nutritionally rich medium used for bacterial growth

M - Molar concentration

$\min -$ minute

$\mathrm{MPa}$ - megapascal

MW - molecular weight

$\mathrm{NaBr}$ - sodium bromide

$\mathrm{NaOCl}$ - sodium hypochloride

$\mathrm{NaOH}$ - Sodium hydroxide

$\mathrm{OH}$ - hydroxyl

$\mathrm{pH}$ - Potential of Hydrogen, a numerical indicator of how acidic or basic a solution is

PLL - poly-L-lysine

PVDF - Poly(vinylidene fluoride)

SEM - Scanning Electron Microscopy

TEMPO - 2,2,6,6-tetramethylpiperidine-1-oxyl radical

wt\% - weight percentage

XRD - X-Ray Diffraction 


\section{Unit Conversion Factors}

\begin{tabular}{|l|c|l|}
\hline Multiply & By & To Obtain \\
\hline angstroms & 0.1 & nanometers \\
\hline degrees (angle) & 0.01745329 & radians \\
\hline degrees Fahrenheit & $(\mathrm{F}-32) / 1.8$ & degrees Celsius \\
\hline feet & 0.3048 & meters \\
\hline horsepower (550 foot-pounds force per second) & 745.6999 & watts \\
\hline microns & $1.0 \mathrm{E}-06$ & meters \\
\hline pints (US liquid) & 0.473176 & liters \\
\hline pounds (force) & 4.448222 & newtons \\
\hline pounds (force) per foot & 14.59390 & newtons per meter \\
\hline pounds (force) per inch & 175.1268 & newtons per meter \\
\hline pounds (force) per square foot & 47.88026 & pascals \\
\hline pounds (force) per square inch & 6.894757 & kilopascals \\
\hline pounds (mass) & 0.45359237 & kilograms \\
\hline
\end{tabular}




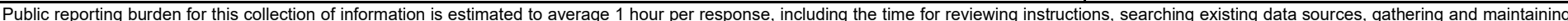

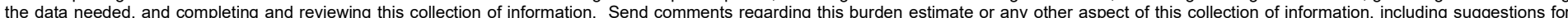

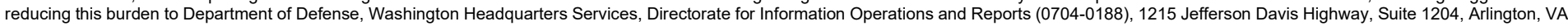

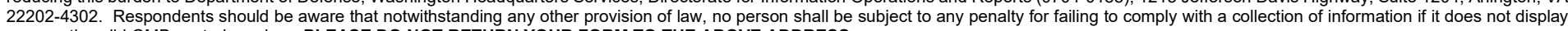
a currently valid OMB control number. PLEASE DO NOT RETURN YOUR FORM TO THE ABOVE ADDRESS.
1. REPORT DATE (DD-MM-YYYY) 2. REPORT TYPE
September 2021 Final
3. DATES COVERED (From - To)

\section{TITLE AND SUBTITLE}

5a. CONTRACT NUMBER

Synthesis and Characterization of Biological Nanomaterial/Poly(vinylidene fluoride)

Composites

\section{5b. GRANT NUMBER}

5c. PROGRAM ELEMENT NUMBER 611102

\section{AUTHOR(S)}

Eftihia Barnes, Jennifer A. Jefcoat, Erik M. Alberts, Hannah R. Peel,

L. Christopher Mimun, J. Paige Buchanan, Xin Guan, Christopher M. Warner,

Kyle L. Klaus, Erin R. Reed-Gore, and Charles A. Weiss Jr. 5d. PROJECT NUMBER

62784

5e. TASK NUMBER

T40

5f. WORK UNIT NUMBER

8. PERFORMING ORGANIZATION REPORT NUMBER

ERDC TR-21-17

See Reverse

\section{PERFORMING ORGANIZATION NAME(S) AND ADDRESS(ES)}

Office of the Assistant Secretary of the Army Acquisition, Logistics and Technology

Washington, DC 20310-0103

\section{DISTRIBUTION / AVAILABILITY STATEMENT}

Approved for public release; distribution is unlimited.

\section{SUPPLEMENTARY NOTES}

62784/T40/Materials and Modeling for Force Protection, "Synthesis and Characterization of Flexible Polymer Composites with Biological Material Enabled Piezoelectricity"

\section{ABSTRACT}

The properties of composite materials are strongly influenced by both the physical and chemical properties of their individual constituents, as well as the interactions between them. For nanocomposites, the incorporation of nano-sized dopants inside a host material matrix can lead to significant improvements in mechanical strength, toughness, thermal or electrical conductivity, etc. In this work, the effect of cellulose nanofibrils on the structure and mechanical properties of cellulose nanofibril poly(vinylidene fluoride) (PVDF) composite films was investigated. Cellulose is one of the most abundant organic polymers with superior mechanical properties and readily functionalized surfaces. Under the current processing conditions, cellulose nanofibrils, as-received and 2,2,6,6tetramethylpiperidine-1-oxyl radical (TEMPO) oxidized, alter the crystallinity and mechanical properties of the composite films while not inducing a crystalline phase transformation on the $\gamma$ phase PVDF composites. Composite films obtained from hydrated cellulose nanofibrils remain in a majority $\gamma$ phase, but also exhibit a small, yet detectable fraction of $\alpha$ and $\beta$ PVDF phases.

$\begin{array}{ll}\text { 15. SUBJECT TERMS } & \text { Nanocellulose } \\ \text { Piezoelectric } & \text { Films } \\ \text { PVDF } & \text { Bacteriophage }\end{array}$

\section{SECURITY CLASSIFICATION OF:}

\section{a. REPORT}

Unclassified

b. ABSTRACT
Unclassified

\section{c. THIS PAGE}

Unclassified
17. LIMITATION OF ABSTRACT

SAR
Composite materials

Nanocomposites (Materials)

Smart materials

Materials--Characterization

\section{NUMBER \\ 19a. NAME OF RESPONSIBLE} OF PAGES

58 PERSON

19b. TELEPHONE NUMBER (include area code) 
PERFORMING ORGANIZATION NAME(S) AND ADDRESS(ES)

Geotechnical and Structures Laboratory

U.S. Army Engineer Research and Development Center 3909 Halls Ferry Road

Vicksburg, MS 39180-6199

Environmental Laboratory

U.S. Army Engineer Research and Development Center 3909 Halls Ferry Road

Vicksburg, MS 39180-6199

HX5 LLC

3530 Manor Drive

Vicksburg, MS 39180-5625

Bennett Aerospace

1100 Crescent Green, Suite 250

Cary, NC 27511-4470 